\title{
On Nonparametric Feature Filters in Electromagnetic Imaging
}

\author{
Jian Zhang ${ }^{1}$ \\ School of Mathematics, Statistics and Actuarial Science, University of Kent, Canterbury, \\ Kent CT2 7NF, UK.
}

\begin{abstract}
Estimation of sparse time-varying coefficients on the basis of time-dependent observations is one of the most challenging problems in statistics. Our study was mainly motivated from magnetoencephalographic neuroimaging, where we want to identify neural activities using the magnetoencephalographic sensor measurements outside the brain. The problem is ill-posed since the observed magnetic field could result from an infinite number of possible neuronal sources. The so-called minimum-variance beamformer is one of data-adaptive nonparametric feature filters to address the above problem in the literature. In this paper, we propose a method of sure feature filtering for a high-dimensional time-varying coefficient model. The new method assumes that the correlation structure of the sensor measurements can be well represented by a set of non-orthogonal variance-covariance components. We develop a theory on the sure screening property of the proposed filters and on when the beamformer-based location estimators are consistent or inconsistent with the true ones. We also derive the lower and upper bounds for the mean filtering errors of the proposed method. The new theory is further supported by simulations and a real data analysis. Keywords: MEG neuroimaging, beamforming, nonparametric feature selection, source screening and reconstruction
\end{abstract}

\footnotetext{
${ }^{1}$ Email: jz79@kent.ac.uk
} 


\section{Introduction}

Suppose that we observe an $n$-dimensional time-course $\mathbf{Y}(t)$ from the model

$$
\mathbf{Y}(t)=\int_{\Xi} \mathbf{x}(r, \eta(r)) \beta(r, t) d r+\varepsilon(t)
$$

where $\Xi$ is a bounded subset of $R^{3}, \beta(r, t)$ is a latent univariate time source of interest at location $r, \mathbf{x}(r, \eta(r))$ is a design vector with nuisance parameter $\eta(r)$, 5 and $\varepsilon(t)$ is a noise. Assume that $\beta(r, t)$ is sparse, i.e., the temporal variability (called power or the marginal variance) $\operatorname{var}(\beta(r,))=$.0 for all $r \in B$ except a few locations (i.e., non-null sources). We want to localize these non-null sources among an infinite number of candidates. Given the limited number of timecourses we observed, the problem is ill-posed and high-dimensional. To simplify

10 it, we discretize the integration, obtaining

$$
\mathbf{Y}(t)=\sum_{k=1}^{p} \mathbf{x}\left(r_{k}, \eta\left(r_{k}\right)\right) \beta\left(r_{k}, t\right)+\varepsilon(t),
$$

where $\Omega=\left\{r_{1}, \ldots, r_{p}\right\}$ is a sieve (or grid) approximation to the source space. The problem becomes a large- $p$-small- $n$ problem. Several new methodologies have been developed for addressing large- $p$-small- $n$ problems in regression settings, including least absolute shrinkage and selection operator (LASSO) (Tibshi15 rani,1996), smoothly clipped absolute deviation (SCAD) (Fan and Li, 2001), and correlation screening (SIS) (Fan and Lv, 2008). Many important theoretical results have also been established recently in selection consistency (e.g.,Meinshausen and Bühmann, 2006; Zhao and Yu, 2006; Zhang, 2010). However, all these works focused on finite dimensional features and are therefore not directly applicable to neuroimaging studies, where features are time series.

In this paper, we propose a nonparametric feature filtering procedure for identifying the sparse coefficients. The proposed procedure is general but was initially designed for magnetoencephalography (MEG) neuroimaging. MEG is a technique for mapping brain activity by measuring magnetic fields produced by electrical currents occurring in the brain, using arrays of superconducting quantum interference sensors (Hamalainen et al., 2010). The MEG neuroimaging 
can be employed to study perceptual and cognitive brain processes, to localize regions affected by pathology, and to determine the function of various parts of the brain. While MEG offers a direct measurement of neural activity with very

30 high temporal resolution, its spatial resolution is relatively low. Concerns over its spatial resolution have raised fundamental issues of methodology and theory. In fact, improving its resolution by virtue of source reconstruction lies at the heart of the entire MEG-based brain mapping enterprise.

In the MEG neuroimaging, $Y_{i}\left(t_{j}\right)$ is the measurement recorded by the MEG 35 sensor $i$ at time $t_{j}$ for $1 \leq i \leq n, 1 \leq j \leq J$, where the time points $t_{j}=j / \Delta$, the number of the time instants $J=b \Delta$ is determined by the time window $b$ and the sampling rate $\Delta$ per second, and the number of the sensors $n$ is of order hundreds. Let $\mathbf{Y}\left(t_{j}\right)$ denote the measurements from all the sensors at time $t_{j}$, which are assumed to be induced by potential sources at locations 40 $r_{k}, 1 \leq k \leq p$ along time-invariant orientations $\eta_{k} \equiv \eta\left(r_{k}, t\right), 1 \leq k \leq p$ in the brain respectively. Let $\beta\left(t_{j}\right)=\left(\beta\left(r_{1}, t_{j}\right), \ldots, \beta\left(r_{p}, t_{j}\right)\right)^{T}$ be the source magnitude vector of these sources at time $t_{j}$ and $\left\{\beta\left(r_{k}, t_{j}\right): 1 \leq j \leq J\right\}$ the source timecourse at $r_{k}$, where the superscript $T$ indicates the matrix transpose. Let $\mathbf{x}_{k}$ be the output vector of these sensors that would be induced by a unit-magnitude

45 source located at $r_{k}$ along orientation $\eta_{k}$ and $\mathbf{X}=\left(\mathbf{x}_{1}, \ldots, \mathbf{x}_{p}\right)$. As a special case of model (1), the sensor measurements $\mathbf{Y}\left(t_{j}\right)$ may be modeled as

$$
\mathbf{Y}\left(t_{j}\right)=\sum_{k=1}^{p} \mathbf{x}_{k} \beta\left(r_{k}, t_{j}\right)+\varepsilon\left(t_{j}\right)
$$

where $\mathbf{x}_{k}=l\left(r_{k}\right) \eta_{k}, l\left(r_{k}\right) \in R^{n \times 3}$ (called lead field matrix at location $r_{k}$ ) is derived from a forward physical model of the brain, and $\varepsilon\left(t_{j}\right)$ is the noise vector of the $n$ sensors at time $t_{j}$ (Sarvas, 1987). To search for unknown sources,

50 a neural activity index for each grid point in the sieve is calculated, creating a source map of brain activity. Important sources can be then identified by filtering the source map. The accuracy of the filtering depends on the sieve size $p$, and the spatial and temporal dimensions of the MEG measurements (i.e., the number of sensors and the number of time instants). In practice, the sieve size ${ }_{55} p$ is often set to a value much larger than $n$. 
The minimum-variance beamforming, a data-adaptive filtering approach to the above source localization problem has been widely used in neuroimaging. In the approach, one scans the source space through a feature space with a series of filters; each is tailored to a particular area of feature (called pass-band) and 60 resistant to confounding effects originating from other areas (called stop-band) (van Veen et al.,1997; Robinson and Vrba, 1998). The scalar minimum variance beamforming aims to estimate the source power at the location $r_{k}$ by minimizing the sample variance of the projected data $w^{T} \mathbf{Y}\left(t_{j}\right), 1 \leq j \leq J$ with respect to the weighting vector $w$, subject to the constraint $w^{T} \mathbf{x}_{k}=1$. In the scalar

65 minimum-variance beamformer, the pass-band is defined by linearly weighting sensor arrays with the constraint $w^{T} \mathbf{x}_{k}=1$, while the stop-band is realized via minimizing the variance of the projected data. The estimated power can be normalized to produce a power map over a given temporal window while the beamformer projected data can provide time course information at each source.

70 We rank these candidate sources by their powers and filter out noisy ones by thresholding.

In recent years, a number of simulation studies and theoretical studies have been conducted to evaluate the performance of a beamformer (e.g., Brookes et al., 2008; Sekihara and Nagarajan, 2010). Despite of this, several issues re75 main to be addressed. Firstly, there is no rigorous statistical theory available to allow one to examine when the estimated source time-courses are consistent with the true ones. In particular, when there are multiple sources, the accuracy is compromised by confounding effects of multiple sources. It is natural to ask when a beamformer will breakdown in presence of multiple sources and how this effect is determined by the spatial and temporal dimensions of a beamformer. Secondly, it is largely unknown in the literature when the beamformer-based filtering procedure can recover the true sources with an overwhelming probability, although a sure filtering procedure for ordinary linear regression models has been developed by Fan and Lv (2008).

85 To address these issues, we propose a beamformer filtering procedure which is based on the thresholded sensor covariance estimator. The objective of the 
procedure is to identify a set of sources from sparse source model (2), which have nonzero powers or power increases relative to a reference. We develop a sure filtering theory for the proposed procedure under certain conditions. We

90 show that if the true sources are not too close to each other and if $n$ and $J$ are large enough, then these sources can be recovered in a probability tending to one. Furthermore, we provide mean error bounds for source localization, power estimation and time-course estimation in the procedure. We conduct simulation studies and a real data analysis to assess the performance of the 95 proposed procedure.

The paper is organized as follows. The details of the new beamforming methodology are provided in Sections 2 and 4. The asymptotic properties of the proposed procedure are investigated in Section 3. The simulation results and a real MEG data analysis are presented in Section 5. The conclusions are made in Section 6. The proof of Theorem 1 is deferred to the Appendix. The part of numerical results and the proofs of the lemmas, the proposition and the other theorems can be found in the on-line supplementary material.

\section{Methodology}

In this section we propose a new filtering procedure which uses the thresholded sensor covariance estimator.

\subsection{Estimation of sensor covariance matrix}

Suppose that $\left(\mathbf{Y}\left(t_{j}\right): 1 \leq j \leq J\right)$ and $\mathbf{X}=\left(\mathbf{x}_{1}, \ldots, \mathbf{x}_{p}\right)$ are randomly sampled from the model (2). Namely,

$$
\mathbf{Y}\left(t_{j}\right)=\mathbf{X} \beta\left(t_{j}\right)+\varepsilon\left(t_{j}\right), \quad 1 \leq j \leq J
$$

where $\mathbf{x}_{k}=l\left(r_{k}\right) \eta_{k}$ is the composite lead field vector at location $r_{k}$ along orientation $\eta_{k}, 1 \leq k \leq p$.

To make the above model identifiable, we assume the following condition.

Condition (A1): The source processes $\{\beta(t)\}$ and the noise process $\{\varepsilon(t)\}$ are stationary with $E[\beta(t)]=E[\varepsilon(t)]=0$. These two processes are uncorrelated 
with each other. The sources $\left\{\beta_{k}(t)\right\}, 1 \leq k \leq p$ are also uncorrelated with each other.

Under model (21) and condition (A1), if the noises are uncorrelated across the sensors and white, then the sensor covariance matrix can be expressed as

$$
C=\mathbf{X} \operatorname{cov}\left(\beta\left(t_{j}\right)\right) \mathbf{X}^{T}+\operatorname{cov}\left(\varepsilon\left(t_{j}\right)\right)=\sum_{k=1}^{p} \gamma_{k} \mathbf{x}_{k} \mathbf{x}_{k}^{T}+\sigma_{0}^{2} I_{n},
$$

where $\gamma_{k}$ denotes the marginal variance (i.e., power) of the $k$-th time-source, $\sigma_{0}^{2}$ is the background noise level and $I_{n}$ is an $n \times n$ identity matrix.

The sensor covariance is traditionally estimated by its sample version,

$$
\hat{C}=\left(\hat{c}_{i j}\right)=\frac{1}{J} \sum_{j=1}^{J}\left(\mathbf{Y}\left(t_{j}\right)-\overline{\mathbf{Y}}\right)\left(\mathbf{Y}\left(t_{j}\right)-\overline{\mathbf{Y}}\right)^{T},
$$

where $\overline{\mathbf{Y}}$ is the sample mean of $\left\{\mathbf{Y}\left(t_{j}\right)\right\}$. It is known that the sample covariance is not a good estimator of the population covariance if its dimension $n$ is large or if the sample covariance is degenerate Bickel and Levina (2008). In MEG neuroimaging, the sample covariance matrix can be nearly singular due to collinearity between nearby voxels, which can have serious effects on estimating the precision matrix used in the source reconstruction. Here, we apply the procedure of Bickel and Levina (2008) to estimate the sensor covariance $C$, which is given by

$$
\hat{C}\left(\tau_{n J}\right)=\left(\hat{c}_{i j}\left(\tau_{n J}\right)\right)
$$

where $\hat{c}_{i j}\left(\tau_{n J}\right)=\hat{c}_{i j} I\left(\left|\hat{c}_{i j}\right| \geq \tau_{n J}\right)$ and $\tau_{n J}$ is a constant changing in $n$ and $J$.

Bickel and Levina (2008) showed that for independent and identically distributed (IID) samples, if one chooses $\tau_{n J}=O(\sqrt{\log (n) / J})$, then under certain regularity conditions the thresholded covariance estimator is consistent with the true one, with the convergence rate of $O_{p}\left(m_{n} \sqrt{\log (n) / J}\right)$, where $m_{n}=\max _{i} \sum_{j=1}^{n} I\left(c_{i j} \neq 0\right)$. Zhang et al.(2014) extended the above result to non IID samples.

\subsection{SAM index}

Source localization can be realized in two steps: Step 1, we construct a sieve $\Phi$ by partitioning the brain into a regular three dimensional grid and calculate 
the source power for each grid point. The size of the sieve is determined by the resolution of the grid. In practice, one often set the resolution level at $1 \mathrm{~cm}$ or $1 \mathrm{~mm}$. These powers generate a power distribution overlaid on a structural image of the subject's brain, creating a brain power map. The beamforming method consider here is termed Synthetic Aperture Magnetometry (SAM) in the literature (Robinson and Vrba, 1998). Step 2, we identify significant sources by thresholding the map. The details are given as follows.

For any grid point $r$ and orientation $\eta$, we define the scaled lead field vector $\mathbf{x}=\mathbf{x}(r, \eta)=l(r) \eta$, where $l(r)$ is the $n \times 3$ lead field matrix. Given $\eta$, we calculate the weighting vector $w(r, \eta)$ by minimizing the variance of $w^{T} \hat{C}\left(\tau_{n J}\right) w$ with respect to $w$, subject to $w^{T} \mathbf{x}=1$. This gives rise to $w(r, \eta)=$ ${ }_{150} \hat{C}\left(\tau_{n J}\right)^{-1} \mathbf{x} / \mathbf{x}^{T} \hat{C}\left(\tau_{n J}\right)^{-1} \mathbf{x}$. The power $\hat{\gamma}(r)$ of the estimated source time-series $\left\{w(r, \eta)^{T} \mathbf{Y}\left(t_{j}\right): 1 \leq j \leq J\right\}$ at $r$ is equal to $1 / \mathbf{x}^{T} \hat{C}\left(\tau_{n J}\right)^{-1} \mathbf{x}$. The orientation is then estimated by maximizing the signal-to-noise ratio

$$
\frac{w(r, \eta)^{T} \hat{C}\left(\tau_{n J}\right) w(r, \eta)}{\left(\sigma_{0}^{2} w(r, \eta)^{T} w(r, \eta)\right)}
$$

or equivalently by maximizing the normalized power

$$
\operatorname{SAM}(\mathbf{x}(r, \eta))=\frac{w(r, \eta)^{T} \hat{C}\left(\tau_{n J}\right) w(r, \eta)}{w(r, \eta)^{T} w(r, \eta)}=\frac{(l(r) \eta)^{T} \hat{C}\left(\tau_{n J}\right)^{-1} l(r) \eta}{(l(r) \eta)^{T} \hat{C}\left(\tau_{n J}\right)^{-2} l(r) \eta}
$$

The above maximization can be done by solving a generalized eigenvalue problem: The optimal orientation $\hat{\eta}(r)$ is the eigenvector associated with the minimum non-zero eigenvalue of the matrix $l(r)^{T} \hat{C}\left(\tau_{n J}\right)^{-2} l(r)$ relative to $l(r)^{T} \hat{C}\left(\tau_{n J}\right)^{-1} l(r)$. Denote $\hat{\mathbf{x}}(r)=\mathbf{x}(r, \hat{\eta}(r))$. We call $\operatorname{sAM}(r)=\operatorname{SAM}(\hat{\mathbf{x}}(r))$ the SAM index of neural activity at $r$. When $r$ is running over the grid points in the brain, $\operatorname{sAm}(r)$ creates a neuronal power map that underlies measured magnetic fields. Zhang et al. 160 (2014) proved that when the underlying true sources are separable in a sense, the SAM index can consistently estimate their powers and therefore identify the true sources from a large number of candidates.

By thresholding the above neuronal power map, we obtain an estimated source set, namely

$$
D_{n}=\left\{r \in \Omega: \operatorname{SAM}(r) \geq \hat{\sigma}_{0}^{2}\left(1+h_{n}\right)\right\},
$$


165 where $\hat{\sigma}_{0}^{2}$ is the estimated background noise level based on a pre-stimulus dataset and $1+h_{n}$ is a pre-selected positive constant or a value estimated from the data. The estimated source time-courses and powers are given by

$$
\begin{aligned}
\Theta_{n} & =\left\{\hat{\mathbf{x}}(r)^{T} \hat{C}\left(\tau_{n J}\right)^{-1} \mathbf{Y}(\cdot) / \hat{\mathbf{x}}(r)^{T} \hat{C}\left(\tau_{n J}\right)^{-1} \mathbf{x}(r): r \in D_{n}\right\} \\
\Gamma_{n} & =\left\{\hat{\gamma}(r): r \in D_{n}\right\}
\end{aligned}
$$

respectively. In this paper, we often let $h_{n}$ increase to infinity with a rate slower than $n$, i.e., $h_{n} / n=o_{p}(1)$. The local peaks of the SAM index over $D_{n}$ give location estimators of the underlying sources. In particular, we are interested in the global maximum of the SAM index, which produces a point estimator called the maximum location estimator for one of the underlying sources.

In practice, the MEG imaging is often run on a subject first without stimulus and then with stimulus. This allows us to calculate the sample covariance $\hat{C}$ 175 for the MEG data with stimulus as well as the sample covariance $\hat{C}_{0}$ for the background noises. The latter can provide an estimator of the background noise level. To make the thresholded sample covariance to be convergent, Zhang et al. (2014) chose $\tau_{n J}=c_{0} \hat{\sigma}_{0}^{2} \sqrt{\log (n) / J}$ with a tuning constant $c_{0}$ and threshold $\hat{C}$ by $\tau_{n J}$, where $\hat{\sigma}_{0}^{2}$ is the minimum diagonal element in $\hat{C}_{0}$. The corresponding SAM index is written as $\operatorname{SAM}_{c_{0}}(r)$. Note that, when $c_{0} \leq 0$, the proposed SAM procedure reduces to the standard SAM implemented in the software FieldTrip. For each value of $c_{0}$, we can apply the proposed SAM procedure to the data and obtain the maximum SAM index

$$
\operatorname{SAM}_{c_{0}}=\max \left\{\operatorname{SAM}_{c_{0}}(r): r \text { in the sieve }\right\}
$$

In both simulations and a real data analysis, Zhang et al.(2014) showed that ${ }_{185} c_{0} \in D_{0}=\{0,0.5,1,1.5,2\}$ had covered its useful range. The issue of how to choose the tuning constant $c_{0}$ has been addressed in Zhang et al.(2014), where they recommended choosing $c_{0}$ so that $\mathrm{SAM}_{c_{0}}$ in (3) attains the maximum. Bickel and Levina (2008) proposed a cross-validation approach for choosing thresholding level in context of covariance matrix estimation. Their method is not directly applicable to the optimization of the SAM mapping as the latter 
involves not only covariance estimation but also other steps. In general, an optimal covariance estimator does not lead to an optimal power map. However, as shown in the following sections, the tuning constant $c_{0}$ has no effect on the asymptotic theory. We will not discuss the issue further and suppress symbol $c_{0}$ in $\operatorname{SAM}_{c_{0}}(r)$ thereafter.

\section{Filter theory}

We present an asymptotic analysis for the proposed procedure when both $n$ and $J$ are tending to infinity. Throughout the paper, we denote by $\|\mathbf{x}\|$ the Euclidean norm of vector $\mathbf{x}$. For an $n \times n$ symmetric matrix $M$, we use $\lambda_{1}(M) \geq$ ${ }_{200} \cdots \geq \lambda_{n}(M)$ for its eigenvalues. Define the operator norm for $M=\left(m_{i j}\right)_{n \times n}$ by $\|M\|=\max _{1 \leq j \leq p}\left|\lambda_{j}(M)\right|$. It is well-known that $\|M\| \leq \max _{i} \sum_{j=1}^{n}\left|m_{i j}\right|$.

\subsection{Reparametrization}

To simplify the derivation, assuming that $l\left(r_{k}\right) \eta_{k} \neq 0,1 \leq k \leq p$, we reparametrize the model (2) as follows:

$$
\mathbf{Y}\left(t_{j}\right)=\left(\tilde{\mathbf{x}}_{1}, \ldots, \tilde{\mathbf{x}}_{n}\right)\left(\begin{array}{c}
\tilde{\beta}_{1}\left(t_{j}\right) \\
\vdots \\
\tilde{\beta}_{p}\left(t_{j}\right)
\end{array}\right)+\varepsilon\left(t_{j}\right),
$$

${ }_{205}$ where $\tilde{\mathbf{x}}_{k}=\sqrt{n} l\left(r_{k}\right) \eta_{k} /\left\|l\left(r_{k}\right) \eta_{k}\right\|$ and $\tilde{\beta}_{k}\left(t_{j}\right)=\left\|l\left(r_{k}\right) \eta_{k}\right\| \beta_{k}\left(t_{j}\right) / \sqrt{n}$. For the notation simplicity, we let $\mathbf{x}_{k}$ and $\beta_{k}\left(t_{j}\right)$ stand for $\tilde{\mathbf{x}}$ and $\tilde{\beta}_{k}\left(t_{j}\right)$ respectively, that is, let

$$
\begin{aligned}
\mathbf{x}_{k} & =\sqrt{n} l\left(r_{k}\right) \eta_{k} /\left\|l\left(r_{k}\right) \eta_{k}\right\|, \\
E\left[\mathbf{Y}\left(t_{j}\right)\right] & =E\left[\beta\left(t_{j}\right)\right]=E\left[\varepsilon\left(t_{j}\right)\right]=0,1 \leq j \leq J, 1 \leq k \leq p
\end{aligned}
$$

in the model (2). The original time-course and power can be recovered by multiplying $\beta\left(r_{k}, t_{j}\right)$ by the scaling factor $\sqrt{n} /\left\|l\left(r_{k}\right) \eta_{k}\right\|$. However, the normalized power index $\operatorname{sam}(r)$ is invariant under this reparametrization. Note that in practice we often see that $\left\|l\left(r_{k}\right) \eta_{k}\right\|^{2} / n=\sum_{i=1}^{n}\left(l_{i}\left(r_{k}\right) \eta_{k}\right)^{2} / n$ is tending to a constant as $n$ is large. See Zhang et al.(2014). 
Assume that the sensor processes are ergodic. Then, the underlying sensor covariance matrix $C_{p}$ can be written as $C_{p}=\sum_{k=1}^{p} \gamma_{k} \mathbf{x}_{k} \mathbf{x}_{k}^{T}+\sigma_{0}^{2} I_{n}$. To build a brain map, we consider an arbitrary location and orientation $(r, \eta)$ in the brain with $l(r) \eta \neq 0$. Let $\mathbf{x}=\mathbf{x}(r, \eta)=\sqrt{n} l(r) \eta /\|l(r) \eta\|$ denote the scaled lead field vector at $r$ along orientation $\eta$. For any two locations $r$ and $r_{y}$, the lead field spatial coherence is defined by $\rho(\mathbf{x}(r, \eta), \mathbf{y})=\mathbf{x}(r, \eta)^{T} \mathbf{y} / n=1-\| \mathbf{x}(r, \eta)-$ $\mathbf{y} \|^{2} /(2 n)$. Note that $\|x(r, \eta)\|=\|y\|=n$. We define the so-called lead field

220 distance between $x(r, \eta)$ and $y$ by $\|\mathbf{x}(r, \eta)-\mathbf{y}\|_{n}=\|\mathbf{x}(r, \eta)-\mathbf{y}\| / \sqrt{n}$. When $\rho(\mathbf{x}(r, \eta), \mathbf{y}) \geq 0$, we have

$$
\begin{aligned}
\frac{1}{2}\|\mathbf{x}(r, \eta)-\mathbf{y}\|_{n}^{2} & =1-\rho(\mathbf{x}(r, \eta), \mathbf{y}) \leq 1-\rho(\mathbf{x}(r, \eta), \mathbf{y})^{2} \\
& \leq 2(1-\rho(\mathbf{x}(r, \eta), \mathbf{y}))=\|\mathbf{x}(r, \eta)-\mathbf{y}\|_{n}^{2} .
\end{aligned}
$$

When $\rho(\mathbf{x}(r, \eta), \mathbf{y})<0$, the above inequalities still hold if we replace $\mathbf{y}$ by $-\mathbf{y}$. So $1-\rho(\mathbf{x}(r, \eta), \mathbf{y})^{2}$ shows how close $(r, \eta)$ is to $\left(r_{y}, \eta_{y}\right)$ in terms of the lead field distances $\|\mathbf{x}(r, \eta)-\mathbf{y}\|_{n}$ and $\|\mathbf{x}(r, \eta)+\mathbf{y}\|_{n}$.

\subsection{Identifiability}

The source identifiability is mainly determined by the lead field matrix. A necessary condition for the source $r_{1}$ being identifiable is that the columns in the matrix are independent of each other (Zhang et al., 2014). That is, we assume the following condition.

Condition (A2): For any three different locations $r, r_{1}$ and $r_{2}$ in the brain, the columns in the matrix $\left(l(r), l\left(r_{1}\right), l\left(r_{2}\right)\right)$ are linearly independent.

\subsection{Convergence of sensor covariance estimator}

Following Bickel and Levina (2008), Zhang et al.(2014) established the convergence rate of the thresholded sensor covariance estimator when both $n$ and ${ }_{235} J$ are tending to infinity under the following two additional conditions.

Condition (A4): There exist positive constants $\kappa_{1}$ and $\tau_{1}$ such that for any $u>0$ and $t$,

$$
\max _{1 \leq i \leq n} P\left(\left\|\mathbf{y}_{i}(t)\right\|>u\right) \leq \exp \left(1-\tau_{1} u^{\kappa_{1}}\right)
$$


and $\max _{1 \leq i \leq n} E\left\|\mathbf{y}_{i}(t)\right\|^{2}<+\infty$.

In the second one, we assume that the sensor processes are strong mixing. Let $\mathcal{F}_{-\infty}^{0}$ and $\mathcal{F}_{k}^{\infty}$ denote the $\sigma$-algebras generated by $\{\mathbf{y}(t):-\infty \leq t \leq 0\}$ and $\{\mathbf{y}(t): t \geq k\}$ respectively. Define the mixing coefficient

$$
\alpha(k)=\sup _{A \in \mathcal{F}_{-\infty}^{0}, B \in \mathcal{F}_{k}^{\infty}}|P(A) P(B)-P(A B)| .
$$

The mixing coefficient $\alpha(k)$ quantifies the degree of the temporal dependence of the process $\{\mathbf{y}(t)\}$ at lag $k$. We assume that $\alpha(k)$ is decreasing exponentially fast as lag $k$ is increasing.

Condition (A5): There exist positive constants $\kappa_{2}$ and $\tau_{2}$ such that for $k \geq 0$,

$$
\alpha(k) \leq \exp \left(-\tau_{2} k^{\kappa_{2}}\right) .
$$

Write $\tau_{n J}=A \sqrt{\log (n) / J}$, where $A$ is a constant. Let $\bar{y}_{i}$ be the sample mean of the $i$-th sensor as before and

$$
\begin{aligned}
C & =C_{p}=\left(c_{i j}\right), \quad m_{n}=\max _{1 \leq i \leq n} \sum_{j=1}^{n} I\left(c_{i j} \neq 0\right), \\
\hat{c}_{i j} & =\frac{1}{J} \sum_{t=1}^{J}\left(y_{i}(t)-\bar{y}_{i}\right)\left(y_{j}(t)-\bar{y}_{j}\right), \\
\hat{C}\left(\tau_{n J}\right) & =\hat{C}_{p}\left(\tau_{n J}\right)=\left(\hat{c}_{i j} I\left(\left|\hat{c}_{i j}\right| \geq \tau_{n J}\right)\right),
\end{aligned}
$$

where $I(\cdot)$ is the indicator. Let $\kappa_{3}=\max \left\{2\left(2 / \kappa_{1}+1 / \kappa_{2}\right)-1,(4 / 3)\left(1 / \kappa_{1}+\right.\right.$ $\left.\left.1 / \kappa_{2}\right)-1 / 3,1\right\}$. We adopt the following result from Zhang et al.(2014).

250 Proposition 3.1. Under conditions $(A 1) \sim(A 5)$, if $(\log (n))^{\kappa_{3}} / J=o(1)$ and $\tau_{n J} m_{n}=o(1)$ as $n \rightarrow \infty$ and $J \rightarrow \infty$, then $\left\|\hat{C}\left(\tau_{n J}\right)^{-1}-C^{-1}\right\|=O_{p}\left(m_{n} \tau_{n J}\right)$ and $\left\|\hat{C}\left(\tau_{n J}\right)^{-2}-C^{-2}\right\|=O_{p}\left(m_{n} \tau_{n J}\right)$.

In practice, $E[\beta(t)]=0$ may not be true even after a centralization. For example, $\beta(t)$ may have a nonlinear trend $\alpha(t)$, i.e., $\beta(t)$ can be expressed as 255 $\alpha(t)$ plus a random process $\zeta(t)$ with $E[\zeta(t)]=0$. In this situation, we modify condition (A1) as follows. 
Condition (A1'): The process $\zeta(t)$ and the noise process $\varepsilon(t)$ are stationary. The noise process is temporally uncorrelated with $\alpha(t)$ and $\zeta(t)$. The components of $\alpha(t)-\sum_{j=1}^{J} \alpha\left(t_{j}\right) / J$ are orthogonal when $J$ is large enough. The

260 components of $\zeta(t)-\sum_{j=1}^{J} \zeta\left(t_{j}\right) / J$ are asymptotically uncorrelated as $J \rightarrow \infty$. Also $\alpha(t)-\sum_{j=1}^{J} \alpha\left(t_{j}\right) / J$ and $\zeta(t)-\sum_{j=1}^{J} \zeta\left(t_{j}\right) / J$ are asymptotically uncorre-

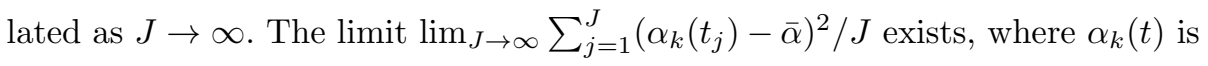
the $k$-th component of $\alpha(t)$.

We re-define the $C$ matrix by letting $\gamma_{k}=\operatorname{var}(\zeta(t))+\lim _{J \rightarrow \infty} \sum_{j=1}^{J}\left(\alpha_{k}\left(t_{j}\right)-\right.$ $\left.{ }_{265} \bar{\alpha}\right)^{2} / J$, where $\alpha_{k}(t)$ is the $k$-th component of $\alpha(t)$ and $\bar{\alpha}=\sum_{j=1}^{J} \alpha\left(t_{j}\right) / J$. Then, Proposition 3.1 still holds under conditions (A1') and (A2) (A5).

\subsection{Bounds for filtering errors}

Under similar conditions to $(\mathrm{A} 1) \sim(\mathrm{A} 5)$, where the true sources are well separated, Zhang et al. (2014) showed the following local consistency result for the SAM index: For non-null true sources,

$\frac{1}{\mathbf{x}_{j}^{T} \hat{C}\left(\tau_{n J}\right)^{-1} \mathbf{x}_{j}}=\gamma_{j}+o_{p}(1), \quad \frac{\mathbf{x}_{j}^{T} \hat{C}\left(\tau_{n J}\right)^{-1} \mathbf{x}_{j}}{\mathbf{x}_{j}^{T} \hat{C}\left(\tau_{n J}\right)^{-2} \mathbf{x}_{j}}=\sigma_{0}^{2}\left(n \gamma_{j} v_{1 j}+1+2 \gamma_{j} v_{2 j}\right)+o_{p}(1)$, where $v_{1 j}$ and $v_{2 j}$ are constants depending on the lead field vectors. In contrast, for null sources,

$$
\frac{1}{\mathbf{x}^{T} \hat{C}\left(\tau_{n J}\right)^{-1} \mathbf{x}}=o_{p}(1), \quad \frac{\mathbf{x}^{T} \hat{C}\left(\tau_{n J}\right)^{-1} \mathbf{x}}{\mathbf{x}^{T} \hat{C}\left(\tau_{n J}\right)^{-2} \mathbf{x}}=\sigma_{0}^{2}+o_{p}(1),
$$

where $\sigma_{0}^{2}$ is the background noise level. In the following, we will present a global screening properties for the SAM index.

275 Suppose that there are only two non-null sources among $p$ potential sources in model (2), which are located at $r_{1}$ and $r_{2}$ with orientations $\eta_{1}$ and $\eta_{2}$ respectively. Let $\mathbf{x}_{1}=l\left(r_{1}\right) \eta_{1}, \mathbf{x}_{2}=l\left(r_{2}\right) \eta_{2}$, and $\psi_{n}=n\left(1-\rho\left(\mathbf{x}_{1}, \mathbf{x}_{2}\right)^{2}\right)$. We assume that the two underlying sources are apart away from each other by a lead field distance of an order larger than $O(1 / n)$ (i.e., $\psi_{n} \rightarrow \infty$ as $n$ tends $\infty$ ). We ${ }_{280}$ choose the two-latent-source model for our study because it is more amenable to theoretical analysis and sharper statements are possible. Although the above 
model is simple, it can provide insight into the more general setting when more than two latent sources exist.

For the simplicity of notations, we let $\Omega$ denote a bounded region of interest in the brain. Let $\mathbf{x}=\mathbf{x}(r, \eta)$ denote the scaled lead field vector at location $r$ along orientation $\eta$ and let $\Phi=\{\mathbf{x} \in B:\|\mathbf{x}\|=n\}$, where $B$ is a set of all the scaled lead field vectors. Let $\hat{\mathbf{x}}(r)=\mathbf{x}(r, \hat{\eta}(r))$ as before. We introduce the following notations.

$$
\begin{aligned}
\rho_{12} & =\rho\left(\mathbf{x}_{1}, \mathbf{x}_{2}\right), \quad \psi_{n}=n\left(1-\rho_{12}^{2}\right), \\
\rho\left(\mathbf{x}, \mathbf{x}_{1}, \mathbf{x}_{2}\right) & =\rho\left(\mathbf{x}, \mathbf{x}_{2}\right)-\rho\left(\mathbf{x}, \mathbf{x}_{1}\right) \rho_{12}, \quad \rho\left(\mathbf{x}, \mathbf{x}_{2}, \mathbf{x}_{1}\right)=\rho\left(\mathbf{x}, \mathbf{x}_{1}\right)-\rho\left(\mathbf{x}, \mathbf{x}_{2}\right) \rho_{12}, \\
\delta_{n}(r \mid 12) & =n \rho\left(\hat{\mathbf{x}}(r), \mathbf{x}_{1}, \mathbf{x}_{2}\right), \quad \delta_{n}(r \mid 21)=n \rho\left(\hat{\mathbf{x}}(r), \mathbf{x}_{2}, \mathbf{x}_{1}\right), \\
\zeta_{n}(r \mid 1) & =n\left(1-\rho\left(\hat{\mathbf{x}}(r), \mathbf{x}_{1}\right)^{2}\right), \quad \zeta_{n}(r \mid 2)=n\left(1-\rho\left(\hat{\mathbf{x}}(r), \mathbf{x}_{2}\right)^{2}\right) \\
\kappa_{n}(r \mid 12) & =\zeta_{n}(r \mid 1)-\delta_{n}(r \mid 12)^{2} / \psi_{n}, \quad \kappa_{n}(r \mid 21)=\zeta_{n}(r \mid 2)-\delta_{n}(r \mid 21)^{2} / \psi_{n}, \\
\delta_{n}(\mathbf{x} \mid 12) & =n \rho\left(\mathbf{x}, \mathbf{x}_{1}, \mathbf{x}_{2}\right), \quad \delta_{n}(\mathbf{x} \mid 21)=n \rho\left(\mathbf{x}, \mathbf{x}_{2}, \mathbf{x}_{1}\right), \\
\zeta_{n}(\mathbf{x} \mid 1) & =n\left(1-\rho\left(\mathbf{x}, \mathbf{x}_{1}\right)^{2}\right), \quad \zeta_{n}(\mathbf{x} \mid 2)=n\left(1-\rho\left(\mathbf{x}, \mathbf{x}_{2}\right)^{2}\right) \\
\kappa_{n}(\mathbf{x} \mid 12) & =\zeta_{n}(\mathbf{x} \mid 1)-\delta_{n}(\mathbf{x} \mid 12)^{2} / \psi_{n}, \quad \kappa_{n}(\mathbf{x} \mid 21)=\zeta_{n}(\mathbf{x} \mid 2)-\delta_{n}(\mathbf{x} \mid 21)^{2} / \psi_{n} .
\end{aligned}
$$

Note that $\kappa_{n}(r \mid 12)$ gauges the closeness of $\mathbf{x}(r)$ to $\mathbf{x}_{1}$ adjusted by the interference from $\mathbf{x}_{2}$ and $\kappa_{n}(r \mid 21)$ gauges the closeness of $\mathbf{x}(r)$ to $\mathbf{x}_{2}$ adjusted by the interference from $\mathbf{x}_{1}$. By definition, $\kappa_{n}(r \mid 12) \rightarrow 0$ when $r \rightarrow r_{1}$ and $\kappa_{n}(r \mid 21) \rightarrow 0$ when $r \rightarrow r_{2}$. By Lemma 6.1 in the Appendix, under condition $(\mathrm{A} 2), \kappa_{n}(r \mid 12) \geq 0$ and $\kappa_{n}(r \mid 21) \geq 0$. Therefore, $\delta_{n}(r \mid 12) \leq\left(\zeta_{n}(r \mid 1)+\psi_{n}\right) / 2$ and $\delta_{n}(r \mid 21) \leq\left(\zeta_{n}(r \mid 2)+\psi_{n}\right) / 2$. Here, the following regularity condition is imposed on the lead field matrix, which states that the lead field distance $\zeta_{n}(r \mid 1)$ $\left(\zeta_{n}(r \mid 2)\right)$ and the adjusted lead field distance $\kappa_{n}(r \mid 12)\left(\kappa_{n}(r \mid 21)\right)$ are of the same rate of convergence as $n \rightarrow \infty$. For any constants $a_{n} \rightarrow \infty$ and $b_{n}=O(1)$, let

$$
\begin{aligned}
\Phi_{2} & =\left\{\mathbf{x} \in \Phi:\|\mathbf{x}\|=n, \zeta_{n}(\mathbf{x} \mid k) \geq a_{n}, k=1,2\right\} . \\
\Phi_{3 \mid k} & =\left\{\mathbf{x} \in \Phi:\|\mathbf{x}\|=n, \psi_{n} \zeta_{n}(\mathbf{x} \mid k) \geq a_{n}, \zeta_{n}(\mathbf{x} \mid k) \leq b_{n}\right\}, k=1,2 .
\end{aligned}
$$


Condition (A6): If $\psi_{n} \rightarrow \infty$, then there is a constant $0<c_{12}<1$ such that

$$
\sup _{\mathbf{x} \in \Phi_{3 \mid 1}} \kappa_{n}(\mathbf{x} \mid 12) / \zeta_{n}(\mathbf{x} \mid 1) \geq c_{12}
$$

If $\psi_{n} \rightarrow \infty$, then there is a constant $0<c_{21}<1$ such that

$$
\sup _{\mathbf{x} \in \Phi_{3 \mid 2}} \kappa_{n}(\mathbf{x} \mid 21) / \zeta_{n}(\mathbf{x} \mid 2) \geq c_{21}
$$

300 If $\psi_{n} \rightarrow \infty$, then

$$
\sup _{\mathbf{x} \in \Phi_{2}} \kappa_{n}(\mathbf{x} \mid 12) \rightarrow \infty, \quad \sup _{\mathbf{x} \in \Phi_{2}} \kappa_{n}(\mathbf{x} \mid 21) \rightarrow \infty
$$

For any positive constants $a_{n} \rightarrow \infty$ and $b_{n}=O(1)$ and for $k=1,2$, define

$$
S_{1 \mid k}=\left\{r \in \Omega: \psi_{n} \zeta_{n}(r \mid k) \leq a_{n}\right\}, \quad S_{2 \mid k}=\left\{r \in \Omega: \psi_{n} \zeta_{n}(r \mid k) \leq b_{n}\right\}
$$

which is an $O\left(1 /\left(n \psi_{n}\right)\right)$-lead field neighborhood of the source $r_{k}$. The following theorem implies that with an overwhelming probability, $D_{n}$ includes $S_{2 \mid k}, k=1,2$ as two sub-sets, while being hold within the $O\left(a_{n} /\left(n \psi_{n}\right)\right)$-lead 305 field neighborhoods of the true sources.

Theorem 3.1. Under conditions $(A 1) \sim(A 6)$, if $(\log (n))^{\kappa_{3}} / J=o(1), n \sqrt{\log (n) / J}=$ $o(1), h_{n} / \psi_{n}=o_{p}(1)$, and $\psi_{n} /\left(h_{n} a_{n}\right)=o_{p}(1)$, then as $n \rightarrow \infty$ and $J \rightarrow \infty$,

$$
P\left(\left(S_{2 \mid 1} \cup S_{2 \mid 2}\right) \subseteq D_{n} \subseteq\left(S_{1 \mid 1} \cup S_{1 \mid 2}\right)\right) \rightarrow 1,
$$

and

$$
S A M(r)= \begin{cases}\frac{\gamma_{1} \psi_{n}\left(1+O\left(1 / \psi_{n}\right)\right)}{1+\gamma_{1}^{2}\left(\psi_{n} \zeta_{n}(\mathbf{X} \mid 1)-\delta_{n}(\mathbf{X} \mid 12)^{2}\right) / \sigma_{0}^{4}}, & r \in S_{2 \mid 1}, \\ \frac{\gamma_{2} \psi_{n}\left(1+O\left(1 / \psi_{n}\right)\right)}{1+\gamma_{2}^{2}\left(\psi_{n} \zeta_{n}(\mathbf{X} \mid 2)-\delta_{n}(\mathbf{X} \mid 21)^{2}\right) / \sigma_{0}^{4}}, & r \in S_{2 \mid 2} .\end{cases}
$$

Remark 3.1. Theorem 3.1 suggests that by high enough thresholding (i.e., choosing $h_{n} \rightarrow \infty$ in probability), we are able to detect two contiguous groups of active locations, $S_{2 \mid 1}$ and $S_{2 \mid 2}$, where the $S A M$ index asymptotically attains the local maximums at the true source locations. For all but a small set of $r$ in $D_{n}$, we have the following inequalities $O\left(1 /\left(n \psi_{n}\right)\right) \leq \min \left\{\left|1-\rho\left(\hat{\mathbf{x}}(r), \mathbf{x}_{1}\right)^{2}\right|\right.$, $\left.\left|1-\rho\left(\hat{\mathbf{x}}(r), \mathbf{x}_{2}\right)^{2}\right|\right\} \leq O\left(a_{n} /\left(n \psi_{n}\right)\right)$ for the lead field distance from $r$ to $\left\{r_{1}, r_{2}\right\}$. 
We define the mean errors of the power and the time-course estimation by $\operatorname{MER}\left(\Gamma_{n}\right)=\sup _{r \in D_{n}} \min \left\{\left|\hat{\gamma}(r)-\gamma_{1}\right|,\left|\hat{\gamma}(r)-\gamma_{2}\right|\right\}$ and $\operatorname{MeR}\left(\Theta_{n}\right)=\max \{|| \theta-$ $\left.\beta_{1}\|\wedge\| \theta-\beta_{2} \|: \theta \in \Theta_{n}\right\}$ respectively, where $a \wedge b=\min \{a, b\}$, and

$$
\left\|\theta-\beta_{k}\right\|^{2}=\frac{1}{J} \sum_{j=1}^{J}\left(\theta\left(t_{j}\right)-\beta_{k}\left(t_{j}\right)\right)^{2}, \quad k=1,2 .
$$

We have the following theorem on the mean errors.

Theorem 3.2. Under the conditions in Theorem 3.1, we have

$$
\begin{aligned}
\operatorname{MER}\left(\Gamma_{n}\right) & =O_{p}\left(a_{n} / \psi_{n}+n \sqrt{\log (n) / J}\right) \\
\operatorname{MER}\left(\Theta_{n}\right) & =O_{p}\left(\psi_{n}^{-1 / 2}+n \sqrt{\log (n) / J}+\sqrt{a_{n} /\left(n \psi_{n}\right)}\right) .
\end{aligned}
$$

Remark 3.2. If two underlying sources are well separated from each other in the sense that $1-\rho_{12}^{2} \geq A / n^{\alpha}$ with $0 \leq \alpha<1$ and $A>0$, and if letting $h_{n}=O_{p}\left(n^{1-\alpha} /(\log (\log (n)))\right.$ and $a_{n}=n^{\alpha}(\log (\log (n)))^{2}$, then we have $h_{n} / \psi_{n}=o_{p}(1)$ and $\psi_{n} /\left(h_{n} a_{n}\right)=o_{p}(1)$. The above mean errors are bounded by $O_{p}\left((\log (\log (n)))^{2} / n^{1-2 \alpha}+n \sqrt{\log (n) / J}\right)$ and $O_{p}\left(1 / n^{(1-\alpha) / 2}+n \sqrt{\log (n) / J}\right)$ respectively.

Remark 3.3. Note that the SAM index is asymptotically flat in the $O\left(1 /\left(n \psi_{n}\right)\right)$ neighborhoods of the true source locations. This suggests that the sieve grid points should be distributed with a spacing of order $O\left(1 /\left(n \psi_{n}\right)\right)$.

We now turn to the case where two underlying sources are not well separated 330 in the sense that $\psi_{n}=O(1)$. For any positive constants $b_{n}=O(1)$, define sets $S_{4 \mid k}=\left\{r \in \Omega: \zeta_{n}(r \mid k) \leq b_{n}\right\}$ for $k=1,2$.

Theorem 3.3. If $\psi_{n} \rightarrow \psi_{0}$ and $n \sqrt{\log (n) / J}=o(1)$ as $n$ and $J$ tend to infinity, then under the conditions in Theorem 3.1, uniformly for $r \in S_{4 \mid 1} \cup S_{4 \mid 2}$, we have

$$
\hat{\gamma}(r)=\left\{\begin{array}{c}
u_{n}(r \mid 1)^{-1}\left(\gamma_{1}+\gamma_{2}\left(1+\psi_{n} \gamma_{1} / \sigma_{0}^{2}\right)\right) \\
\times\left(1+o_{p}(1)\right), \text { when } r \in S_{4 \mid 1}, \\
u_{n}(r \mid 2)^{-1}\left(\gamma_{2}+\gamma_{1}\left(1+\psi_{n} \gamma_{2} / \sigma_{0}^{2}\right)\right) \\
\times\left(1+o_{p}(1)\right), \text { when } r \in S_{4 \mid 2},
\end{array}\right.
$$




$$
\operatorname{SAM}(r)=\left\{\begin{array}{c}
v_{n}(r \mid 1)^{-1} u_{n}(r \mid 1) \sigma_{0}^{4}\left(r_{1}+r_{2}\left(1+r_{1} \psi_{n} / \sigma_{0}^{2}\right)\right) \\
\times\left(1+o_{p}(1)\right), \text { when } r \in S_{4 \mid 1}, \\
v_{n}(r \mid 2)^{-1} u_{n}(r \mid 2) \sigma_{0}^{4}\left(r_{2}+r_{1}\left(1+r_{2} \psi_{n} / \sigma_{0}^{2}\right)\right) \\
\times\left(1+o_{p}(1)\right), \text { when } r \in S_{4 \mid 2},
\end{array}\right.
$$

where

$$
\begin{aligned}
u_{n}(r \mid 1)= & 1+\frac{\gamma_{1} \zeta_{n}(r \mid 1)}{\sigma_{0}^{2}}+\frac{\gamma_{2}}{\sigma_{0}^{2}}\left(\zeta_{n}(r \mid 1)+\psi_{n}-2 \delta_{n}(r \mid 12)\right) \\
& +\frac{\gamma_{1} \gamma_{2}}{\sigma_{0}^{4}}\left(\zeta_{n}(r \mid 1) \psi_{n}-\delta_{n}^{2}(r \mid 12)\right), \\
u_{n}(r \mid 2)= & 1+\frac{\gamma_{2} \zeta_{n}(r \mid 2)}{\sigma_{0}^{2}}+\frac{\gamma_{1}}{\sigma_{0}^{2}}\left(\zeta_{n}(r \mid 2)+\psi_{n}-2 \delta_{n}(r \mid 21)\right) \\
& +\frac{\gamma_{1} \gamma_{2}}{\sigma_{0}^{4}}\left(\zeta_{n}(r \mid 2) \psi_{n}-\delta_{n}^{2}(r \mid 21)\right) . \\
v_{n}(r \mid 1)= & \zeta_{n}(r \mid 1)\left(r_{1}+r_{2}\left(1+r_{1} \psi_{n} / \sigma_{0}^{2}\right)\right)^{2}+r_{2}\left(1+r_{1} \delta_{n}(r \mid 12) / \sigma_{0}^{2}\right) \\
& \times\left(\psi_{n} r_{2}-\delta_{n}(r \mid 12)\left(2\left(r_{1}+r_{2}\right)+r_{1} r_{2} \psi_{n} / \sigma_{0}^{2}\right)\right), \\
v_{n}(r \mid 2)= & \zeta_{n}(r \mid 2)\left(r_{2}+r_{1}\left(1+r_{2} \psi_{n} / \sigma_{0}^{2}\right)\right)^{2}+r_{1}\left(1+r_{2} \delta_{n}(r \mid 21) / \sigma_{0}^{2}\right) \\
& \times\left(\psi_{n} r_{1}-\delta_{n}(r \mid 21)\left(2\left(r_{1}+r_{2}\right)+r_{1} r_{2} \psi_{n} / \sigma_{0}^{2}\right)\right) .
\end{aligned}
$$

335 For any positive constants $a_{n} \rightarrow \infty$, define sets $S_{5 \mid k}=\left\{r \in \Omega: \zeta_{n}(r \mid k) \leq\right.$ $\left.1 / a_{n}\right\}$.

Corollary 3.1. If $\psi_{n} \rightarrow \psi_{0}$ and $n \sqrt{\log (n) / J}=o(1)$ as $n$ and $J$ tend to infinity, then uniformly for $r \in S_{5 \mid 1} \cup S_{5 \mid 2}$, we have

$$
\begin{gathered}
\hat{\gamma}(r)=\left\{\begin{array}{cl}
\left(\gamma_{1}+\frac{\gamma_{2}}{1+\psi_{0} \gamma_{2} / \sigma_{0}^{2}}\right)\left(1+o_{p}(1)\right), \quad r \in S_{5 \mid 1} \\
\left(\gamma_{2}+\frac{\gamma_{1}}{1+\psi_{0} \gamma_{1} / \sigma_{0}^{2}}\right)\left(1+o_{p}(1)\right), \quad r \in S_{5 \mid 2} .
\end{array}\right. \\
\operatorname{SAM}(r)=\left\{\begin{array}{c}
\frac{\sigma_{0}^{2}}{\gamma_{2}^{2} \psi_{0}}\left(\sigma_{0}^{2}+\gamma_{2} \psi_{0}\right)\left(\gamma_{1}+\gamma_{2}\left(1+\gamma_{1} \psi_{0} / \sigma_{0}^{2}\right)\right) \\
\times\left(1+o_{p}(1)\right), \text { when } r \in S_{5 \mid 1}, \\
\frac{\sigma_{0}^{2}}{\gamma_{1}^{2} \psi_{0}}\left(\sigma_{0}^{2}+\gamma_{1} \psi_{0}\right)\left(\gamma_{2}+\gamma_{1}\left(1+\gamma_{2} \psi_{0} / \sigma_{0}^{2}\right)\right) \\
\times\left(1+o_{p}(1)\right), \text { when } r \in S_{5 \mid 2} .
\end{array}\right.
\end{gathered}
$$

340 Remark 3.4. Denote $\alpha^{2}=\delta_{n}^{2}(r \mid 12) /\left(\psi_{n} \zeta_{n}\right)$. In Figures 1 and 2 in the online supplemental material, we plotted the asymptotic SAM derived in Theorem 3.3 
against the asymptotic value of $\zeta_{n}(r \mid 1)$ for various combinations of $\left(\psi_{0}, \alpha^{2}\right)$, where $\psi_{0} \in\{0,0.5,1,2,3,4\}, \alpha^{2} \in\{0,0.0392,0.1584,0.3576,0.4872,0.6368$, $0.8064,0.9960\}$. We assumed that there were two nonzero underlying sources with powers $\gamma_{1}=2$ and $\gamma_{2}=3$ respectively and that the noise level $\sigma_{0}^{2}=1$. Note that $\psi_{0}$ shows how close two underlying sources are to each other, while $\alpha^{2}$ measures the degree of coherence of lead field vectors in the neighborhoods of the underlying sources. Let $\hat{r}$ denote a local peak location in the SAM curve. Let $\zeta$ be the limit of $\zeta_{n}$. If the asymptotic $S A M$ attains the peak at $\zeta>0$, then $\hat{r}$ is asymptotically inconsistent with the true source location $r_{1}$ in the sense that the lead field discrepancy $n\left(1-\rho\left(\mathbf{x}(\hat{r}), \mathbf{x}_{1}\right)^{2}\right)$ is not close to zero. Figures 1 and 2 in the online supplemental material indicate that the consistency of the SAM-based localization depends on the degree of the separateness between the underlying sources as well as the degree of the coherence among $\mathbf{x}(\hat{r}), \mathbf{x}_{1}$, and $\mathbf{x}_{2}$. For example, Figures 1 and 2 show that the SAM peak can occur at nonzero $\zeta$ if $\psi_{0}=0.5$ and $\alpha^{2}>0.1584$, or if $\psi_{0}=1, \alpha^{2}>0.3576$, or if $\psi_{0}=2, \alpha^{2}>0.4872$, or if $\psi_{0}=3, \alpha^{2}>0.63668$, or $\psi_{0}=4, \alpha^{2}>0.8064$. In another word, if the underlying sources are not well separated (i.e., $\psi_{n} \rightarrow \psi_{0}>0$ ), the local peaks in the SAM map may not asymptotically occur at the true source locations.

360 Therefore, in these cases, we are unable to localize these sources even after reducing the threshold $h_{n}$ to a lower level. Furthermore, the above corollary shows that if $\psi_{n} \rightarrow \psi_{0}>0$, then the power estimators are always inconsistent with the true ones due to the signal cancellation between the sources, even when their true positions and orientations are known.

Remark 3.5. Theorems 3.1 3.3 still hold if we replace condition (A1) by condition (A1').

\section{Choice of threshold in mapping}

The main product of the proposed screening procedure is the estimated source set $D_{n}$, whose performance depends on the threshold $1+h_{n}$. We choose 370 the threshold based on the idea of clustering as follows. 
It follows from Theorem 3.1 and Lemma 6.4 in the Appendix that if the underlying sources are well separated with moderate or large signal-to-noise ratios, then the values of $\operatorname{SAm}(r) / \hat{\sigma}_{0}^{2}$ at non-source locations are likely to be wandering at around 1, whereas the values of $\operatorname{sAM}(r) / \hat{\sigma}_{0}^{2}$ in neighborhoods of source locations are typically much larger than 1 . Therefore, the values of $\operatorname{sAm}(r) / \hat{\sigma}_{0}^{2}$ can be grouped into two general clusters, one of which is a noise cluster. The positions of the peaks in the clusters give a clue to the source locations. The lower bound of the $\operatorname{SAM}(r) / \hat{\sigma}_{0}^{2}$ peaks in the non-noise clusters provides a natural estimate of the threshold value for the screening. In our implementation, we refine the above idea by taking into account the following structure of the SAM mapping, i.e., the grouping of the SAM values often be affected by the spatial coherence of grid points. In neuroimaging, it is a common practice to reduce the complexity of the problem by first partitioning the grid points into a number of transverse slices along the $z$-axis of the brain. Except the noise 385 slices, the SAM values in each of brain slices except noisy ones often peak toward a source location. Therefore, these peak values can be used as summary statistics for the non-noise group. In practice, we need to calculate the peak values $s_{1}, \cdots, s_{k_{0}}$ for these slices. Sorting these values in descending order, we have order statistics $s_{(1)}, \cdots, s_{\left(k_{0}\right)}$. Plotting these ordered values against their 390 indices $k=1, \cdots, k_{0}$ gives rise to a scree plot, where the peaks are decreasing as one moves to the right. When the dropping rate changes, the curve in the plot has an elbow or a change-point. See Figure 3 in the online supplemental material for a typical scree plot in our simulations conducted in the next section. The peak values around the change-point can be used to estimate $1+h_{n}$. For simplicity, we estimate the change-point by searching for $k$ at which $s_{(k+1)}-s_{(k)}$ attains the maximum in our simulations. A more refined but time-consuming way to determine the change-point would be to employ the gap-statistic based procedure, where we could adjust for spatially varying baseline-effects by using some reference distribution derived from multiple trial information (Tibshinari ${ }_{400}$ et al., 2001). See the real data analysis in the next section for the details. 


\section{Numerical results}

\subsection{Simulation results}

In this section, we examined the finite-sample performance of the proposed beamformer procedure under various scenarios via simulations, where $n \sqrt{\log (n) / J}$

405 is not small. We need more notations as follows. For any estimator $\hat{r}_{1}$ of source location $r_{1}$, we denote by $E\left|\hat{r}_{1}-r_{1}\right|$ the mean localization bias, where $\left|\hat{r}_{1}-r_{1}\right|$ is the $L_{1}$-distance between $\hat{r}_{1}$ and $r_{1}$. We denote by $\rho_{\max }$ the maximum coherence between two locations $r_{1}$ and $r_{2}$ with

$$
\rho_{\max }\left(r_{1}, r_{2}\right)=\max _{\left\|\eta_{1}\right\|=1,\left\|\eta_{2}\right\|=1} \frac{\left(l\left(r_{1}\right) \eta_{1}\right)^{T} l\left(r_{2}\right) \eta_{2}}{\left\|l\left(r_{1}\right) \eta_{1}\right\| \cdot\left\|l\left(r_{2}\right) \eta_{2}\right\|} .
$$

We assessed the behavior of the beamformer-based maximum location estimator by its mean $L_{1}$-distance to the underlying sources. Similarly, the accuracy of the estimated source set $D_{n}$ derived from the proposed screening can be measured by its closeness to the underlying sources, namely $d\left(D_{n},\left\{r_{1}, r_{2}\right\}\right)=$ $\max _{r_{0} \in\left\{r_{1}, r_{2}\right\}} \min _{r \in D_{n}}\left|r-r_{0}\right|$, and by its size. Good $D_{n}$ requires a trade-off between the size and the closeness to the underlying sources. By using the simulations, we attempt to answer the following questions: (1) How does the tuning constant $c_{0}$ improve the performance of the SAM screening procedure? (2) Is the screening procedure a valuable complement to the SAM-based maximum location estimator? (3) To what extent will the performance of the proposed procedure deteriorate by source cancellations and correlations? (4) What is the performance of the proposed procedure when the assumptions we made in Theorems 3.1 3 are invalid?

We simulated a 184-sensor MEG system (CTF/VSM) using a real adult human subject head shape which was approximated by multiple local spheres and downloaded at http://fieldtrip.fcdonders.nl/download. We constructed $p=$ 2905 regular 3-D grid points of resolution $1 \mathrm{~cm}$ within the head. These candidate source positions were aligned with the axes of the head coordinate system. A lead field matrix $L(184 \times 8715)$ between the $n=184$ sensors and the 2905 grid points was then calculated by using the software FieldTrip. Note that 
the sieve size $p$ is determined by the resolution. If we refine the sieve so that ${ }_{430}$ it is of resolution $1 \mathrm{~mm}$, we can obtain 10 times more grid points. Then, the computation in beamforming is too time-consuming to be realized in an ordinary PC.

We assumed that there were two non-zero sources $\theta_{1}(t)$ and $\theta_{2}(t)$, which were located at $r_{1}=(5,-2,8)^{T}$ and $r_{2}=(-5,-2,8)$ in the auditory area with the dumping cosine patterns $\theta_{1}(t)=\eta_{1} \beta_{1}(t)$ and $\theta_{2}(t)=\eta_{2} \beta_{2}(t)$ respectively. The $L_{1}$ distance and the maximum coherence between the two sources are 10 $\mathrm{cm}$ and 0.3632 respectively. Here,

$$
\begin{aligned}
\eta_{1}= & \left(\frac{10}{\sqrt{102}}, \frac{1}{\sqrt{102}}, \frac{1}{\sqrt{102}}\right)^{T}, \quad \eta_{2}=(1,0,0), \\
a_{1}= & \sqrt{102}, \quad a_{2}=8, \\
\beta_{k}(t) / a_{k}= & z_{k}(t) \\
+ & \left\{\begin{array}{l}
\exp \left(-\left(t-\frac{m_{k}}{m_{0}}-\pi\right)^{2}\right) \\
\times\left(1-\frac{\left(t-m_{k} / m_{0}-m_{0} / 60000\right)^{2}}{\left(1-1 / m_{0}+m_{0} / 60000\right)^{2}}\right) \\
\times \cos \left(2 \pi f_{k}\left(t-\frac{m_{k}}{m_{0}}\right)-\pi\right), \\
\frac{m_{k}}{m_{0}} \leq t \leq 1+\frac{\left(m_{k}-1\right)}{m_{0}} \\
0,
\end{array} \quad\right. \text { Otherwise, }
\end{aligned}
$$

where $m_{0}, m_{k}$ and $f_{k}$ are two factors related to time-shifts and frequencies of the cosine waves, and $z_{k}(t) \sim \operatorname{AR}(1)$, i.e., $z_{k}(t)=0.2 z_{k}(t-1)+e(t),\{e(t)\}$ is ${ }_{440}$ a white noise process with mean 0 and variance $0.1^{2}$. Note that $E\left[\beta_{k}(t)\right] \neq 0$. Therefore, condition (A1) may not hold true. Let $J=2 m_{0}$. We considered three scenarios: $(1)\left(m_{1}, m_{2}\right)=m_{0} \times(1 / 10,1 / 2)$ and $\left(f_{1}, f_{2}\right)=(1,1)$; $\left(m_{1}, m_{2}\right)=m_{0} \times(1 / 8,1 / 4)$ and $\left(f_{1}, f_{2}\right)=(1,3) ;(3)\left(m_{1}, m_{2}\right)=m_{0} \times(1 / 8,1 / 8)$ and $\left(f_{1}, f_{2}\right)=(1,1)$. We generated a pair of signals for each scenario with $m_{0}=$ ${ }_{445} 240,500,1000$, and 1500 respectively. As examples, the pairs of signal curves in these scenarios for $m_{0}=500$ were plotted in Figure 3 in the online supplemental material. The temporal correlation coefficients between these paired curves are $-0.462,0.043,0.706$ respectively. These paired curves stand for the three cases, where the paired sources are negatively correlated, positively and weak 
450 correlated, and positively and strong correlated respectively. As $n \sqrt{\log (n) / J}$ is not small in these scenarios, the conditions for Theorem 3.1 do not hold.

The sensor measurements at $t$ follow the model

$$
Y(t)=l\left(r_{1}\right) \eta_{1} \beta_{1}(t)+l\left(r_{2}\right) \eta_{2} \beta_{2}(t)+\varepsilon(t), \quad 0 \leq t \leq 1
$$

where $\varepsilon(t)$ is the sensor noise vector. Let the time window width $w=2$. That is, the sensors were measured at the time instants $t_{k}=2 k / J, k=0,1,2, \ldots, J-1$. The signal strength (SS) in the sensor space was defined by

$$
\mathrm{SS}=\sqrt{\sum_{k=0}^{J-1} \frac{\left\|l\left(r_{1}\right) \eta_{1} \beta_{1}\left(t_{k}\right)\right\|^{2}}{J}} .
$$

For each $k$, we sampled $N_{n k}$ from an $n$-dimensional standard Normal and set $\varepsilon\left(t_{k}\right)=\mathrm{SS} \times N_{n k} / \sqrt{\mathrm{SNR}}$. Here, we considered three values of SNR, 1.5625, 1 , and 0.01 , standing for the cases with moderate signals and weak signals respectively.

For each combination of SNR, $\left(m_{1}, m_{2}\right)$ and $\left(f_{1}, f_{2}\right)$, we independently generated 50 datasets of $\left\{\left(Y\left(t_{k}\right), 0 \leq k \leq J-1\right\}\right.$ paired with $\left\{\varepsilon\left(t_{k}\right), 0 \leq k \leq J-1\right\}$ by (4). Here, we imitated the practical situation, where the MEG imaging was run on a subject first without stimulus and then with stimulus. The former provides an estimator of the background noise level. For each dataset, we calcu-

465 lated the sample covariance $\hat{C}$ of $\left\{\left(Y\left(t_{k}\right), 0 \leq k \leq J-1\right\}\right.$ and the corresponding sample covariance $\hat{C}_{0}$ of the background noises. We set $\tau_{n J}=c_{0} \hat{\sigma}_{0}^{2} \sqrt{\log (n) / J}$ with the tuning constant $c_{0}$ and thresholded $\hat{C}$ by $\tau_{n J}$, where $\hat{\sigma}_{0}^{2}$ is the minimum diagonal element in $\hat{C}_{0}$. We considered four values for $c_{0}$ : $0,0.5,1.5$, and 2. Note that, when $c_{0}=0$, the proposed SAM procedure reduces to the standard SAM implemented in the FieldTrip. For each value of $c_{0}$, we applied the proposed SAM procedure to the 50 datasets, where the threshold $1+h_{n}$ is determined by the scree algorithm described in the previous section. For each dataset, we obtained the SAM-based maximum location estimate and selected the source set $D_{n}$ by thresholding. We calculated the average minimum localization biases of the SAM-based maximum location estimates to $r_{1}$ and $r_{2}$ as 
well as the standard errors over these 50 datasets. For each dataset, we also calculated the maximum $L_{1}$-distances from $D_{n}$ to the two underlying sources $r_{1}$ and $r_{2}$ as well as the size of $D_{n}$, where the distance from $D_{n}$ to $r_{k}$ is defined by $d\left(D_{n}, r_{k}\right)=\min \left\{\left|r-r_{k}\right|: r \in D_{n}\right\}$. We calculated the averages and stan480 dard errors of these quantities over these 50 datasets respectively. Note that, when both the mean and variance of the maximum $L_{1}$-distance from $D_{n}$ to the underlying sources are zero, $D_{n}$ recovers all the true sources. By dividing the average size of $D_{n}$ by the total number of candidate sources, 2905, we obtain the average proportion of the candidate sources being selected. The maximum

${ }_{485} L_{1}$-distance from $D_{n}$ to $r_{1}$ and $r_{2}$ measures the approximate coverage of the true sources, while the size of $D_{n}$ implies the false discovery rate. So, given the distance from $D_{n}$ to the true sources, the smaller size the better $D_{n}$ is.

The results, summarized in Table 1 and Tables $1 \sim 2$ in the online supplemental material, show that: (1) Using the thresholded covariance, we can 490 improve the performances of the maximum location estimator and the estimated source set slightly in terms of localization bias when signals are weak. In practice, the preferred values of $c_{0}$ may be lying between 0.5 and 1.5 for weak signals. (2) The performances were robust to the potential source correlations. (3) For strong or moderately strong sources, the estimated source set contained 495 or was very close to the true sources most of the time as the maximum-minimum distances from the estimated source sets to the underlying two sources have an mean 0 and a variance 0 approximately. For weak sources, the estimated source set also had reasonable closeness to the true sources if choosing $c_{0}$ properly. For moderate source signals, the size of $D_{n}$ is around 7, which is extremely tiny, compared to the number of the grids, 2905, indicating that the screening result is very accurate. However, the size of the estimated source set may increase to a few hundreds when the signal-to-noise ratio is low, reflecting that $D_{n}$ contained many false discoveries. In Scenario 3 with $\mathrm{SNR}=0.01$, the SAM-based maximum location estimator has a $L_{1}$-bias ranging from 2 to 5 , suggesting that 505 it has missed the true sources on average. See Table 1 in the online supplemental material. Therefore, the screening procedure may be better than the 
single-point estimator in identifying the underlying sources.

The results, displayed in Tables $3 \sim 6$ in the online supplemental material, demonstrate that if there is only one source, say $\beta_{2}(t)$ in the data, we can estimate the source very accurately either by using the maximum location estimator or by the screening. However, compared to the results in Table 1 and Tables $1 \sim 2$ in the online supplemental material, we can see that if we added another source $\beta_{1}(t)$ to the model, then the coherence between the lead field vectors can have a serious effect on the estimation of source $\beta_{2}(t)$. Sometimes, source $\beta_{2}(t)$ can be completely masked by the interference from source $\beta_{1}(t)$.

In summary, the simulation results suggest that:

- The source interference due to the lead field coherence has sever effects on recovering sources. In fact, as demonstrated in Table 1 and Tables $1 \sim 6$ in the online supplemental material, without the interference, we can accurately localize the source by using the SAM-based maximum location estimator or by using the screening. In particular, the size of the selected set $D_{n}$ can be substantially inflated by source interference. In general, when the sample size $J$ is larger than 1000 , the screening procedure is extremely good at detecting sources with moderate or strong signals.

- The proposed screening procedure offers a better source estimation than a point estimator such as the maximum location estimator in the presence of multiple sources. Although the accuracy of the proposed source screening can be affected by the signal-to-noise ratio and by the degree of source interference, it is robust to the underlying source correlations. On average, the accuracy can be improved by choosing the tuning constant $c_{0}=0.5$, or 1 , or 1.5 , or 2 in the sensor covariance estimation when the SNR is low. However, when the SNR is not low, it may be better to choose $c_{0}=0$. The results also imply that there is not a universal choice of $c_{0}$. Rather than choosing a universal one, we should choose $c_{0}$ adaptive to the SAM indices over a range of $c_{0}$. Zhang et al.(2014) provided two of such selection procedures for the maximum location estimator. 
Table 1: Simulation results for the estimated source set $D_{n}$ in the two-source setting. The average maximum $L_{1}$-distance from $D_{n}$ to the underlying sources, $\max \left\{d\left(D_{n}, r_{k}\right): k=1,2\right\}$, and the average size of $D_{n}$ over 50 repetitions are provided for Scenarios 1 to 3 when SNR=0.01 and 1 respectively. The standard errors are given in the parentheses.

\begin{tabular}{|c|c|c|c|c|c|c|}
\hline$J$ & Scen. & $c_{0}=0$ & $c_{0}=0.5$ & $c_{0}=1$ & $c_{0}=1.5$ & $c_{0}=2$ \\
\hline \multicolumn{7}{|c|}{$\mathrm{SNR}=0.01$} \\
\hline & & \multicolumn{5}{|c|}{ Average $\max _{k} d\left(D_{n}, r_{k}\right)$ (Std. error) } \\
\hline 480 & 1 & $1.76(0.45)$ & $2.56(0.669)$ & $2.12(0.536)$ & $6.04(0.885)$ & $11.6(1.00)$ \\
\hline 1000 & 1 & $0.46(0.25)$ & $0.18(0.16)$ & $1.34(0.46)$ & $5.66(0.67)$ & $10.9(0.77)$ \\
\hline 2000 & 1 & $0.04(0.03)$ & $0.02(0.02)$ & $3.3(0.65)$ & $9.04(0.31)$ & $9.26(0.48)$ \\
\hline 3000 & 1 & $0.74(0.34)$ & $0.2(0.18)$ & $1.5(0.5)$ & $7.5(0.53)$ & $8.8(0.43)$ \\
\hline 480 & 2 & $2.02(0.43)$ & $1.88(0.40)$ & $0.40(0.16)$ & $6.1(0.92)$ & $10.3(0.86)$ \\
\hline 1000 & 2 & $1.06(0.37)$ & $0.66(0.31)$ & $2.14(0.53)$ & $4.2(0.67)$ & $10(0.88)$ \\
\hline 2000 & 2 & $0.8(0.33)$ & $0.32(0.22)$ & $1.26(0.42)$ & $6.3(0.63)$ & $9.1(0.68)$ \\
\hline 3000 & 2 & $0.36(0.21)$ & $0(0)$ & $1.14(0.43)$ & $7.3(0.52)$ & $9.02(0.50)$ \\
\hline 480 & 3 & $1.98(0.32)$ & $2.16(0.33)$ & $1.44(0.30)$ & $2.54(0.61)$ & $11.2(0.89)$ \\
\hline 1000 & 3 & $1.56(0.26)$ & $0.96(0.17)$ & $0.3(0.11)$ & $2.52(0.48)$ & $9.78(0.87)$ \\
\hline 2000 & 3 & $1.12(0.17)$ & $0.54(0.13)$ & $0.36(0.09)$ & $2.36(0.46)$ & $8.5(0.79)$ \\
\hline \multirow[t]{2}{*}{3000} & 3 & $0.88(0.13)$ & $0.58(0.12)$ & $0.52(0.17)$ & $3.64(0.54)$ & $8.32(0.76)$ \\
\hline & & \multicolumn{5}{|c|}{ Average size (Std. error) } \\
\hline 480 & 1 & $351(73.7)$ & $414(86)$ & $559(97)$ & $309(75)$ & $141(54)$ \\
\hline 1000 & 1 & $281(83)$ & 449 (104) & $305(82)$ & $138(40)$ & $62(23)$ \\
\hline 2000 & 1 & $269(76)$ & $276(77)$ & $35(5.7)$ & $13(1.2)$ & $48(20)$ \\
\hline 3000 & 1 & $197(58)$ & $235(71)$ & $53(10)$ & $19(2.4)$ & $21(5)$ \\
\hline 480 & 2 & $311(78)$ & $553(103)$ & 707 (95) & $278(64)$ & $144(52)$ \\
\hline 1000 & 2 & $261(72)$ & $509(109)$ & $406(90)$ & $361(76)$ & $143(51)$ \\
\hline 2000 & 2 & $120(34)$ & $281(77)$ & $228(73)$ & $117(43)$ & $68(27)$ \\
\hline 3000 & 2 & $439(100)$ & $318(87)$ & $60(10)$ & $29(7.5)$ & $22(5.3)$ \\
\hline 480 & 3 & $518(91)$ & $536(95)$ & $838(130)$ & $496(82)$ & $128(40)$ \\
\hline 1000 & 3 & $419(94)$ & $631(120)$ & $1008(123)$ & $414(77)$ & $143(39)$ \\
\hline 2000 & 3 & $303(83)$ & $574(114)$ & $490(96)$ & $260(60)$ & $134(33)$ \\
\hline 3000 & 3 & $432(101)$ & $522(110)$ & $387(81)$ & $234(66)$ & $82(18)$ \\
\hline \multicolumn{7}{|c|}{$\mathrm{SNR}=1$} \\
\hline & & \multicolumn{5}{|c|}{ Average $\max _{k} d\left(D_{n}, r_{k}\right)$ (Std. error) } \\
\hline 480 & 1 & $0(0)$ & $0(0)$ & $0.04(0.028)$ & $0.18(0.18)$ & $0.3(0.204)$ \\
\hline 1000 & 1 & $0(0)$ & $0(0)$ & $0.02(0.02)$ & $0.04(0.03)$ & $0.18(0.18)$ \\
\hline 2000 & 1 & $0.02(0.02)$ & $0.02(0.02)$ & $0.04(0.028)$ & $0.02(0.02)$ & $0(0)$ \\
\hline 3000 & 1 & $0(0)$ & $0.06(0.03)$ & $0(0)$ & $0.02(0.02)$ & $0.02(0.02)$ \\
\hline 480 & 2 & $0.06(0.03)$ & $0(0)$ & $0.04(0.03)$ & $0(0)$ & $0.2(0.2)$ \\
\hline 1000 & 2 & $0.02(0.02)$ & $0(0)$ & $0(0)$ & $0(0)$ & $2(0.53)$ \\
\hline 2000 & 2 & $0.06(0.03)$ & $0(0)$ & $0.02(0.02)$ & $0.06(0.03)$ & $0(0)$ \\
\hline 3000 & 2 & $0.04(0.03)$ & $0.02(0.02)$ & $0.02(0.02)$ & $0.02(0.02)$ & $0(0)$ \\
\hline 480 & 3 & $0.02(0.02)$ & $0.06(0.03)$ & $0.3(0.18)$ & $0.94(0.31)$ & $0.26(0.18)$ \\
\hline 1000 & 3 & $0(0)$ & $0.02(0.02)$ & $0.08(0.04)$ & $0.08(0.04)$ & $0.48(0.20)$ \\
\hline 2000 & 3 & $0.06(0.03)$ & $0.06(0.03)$ & $0.02(0.02)$ & $0.08(0.04)$ & $0.1(0.04)$ \\
\hline \multirow[t]{2}{*}{3000} & 3 & $0.02(0.10)$ & $0.10(0.04)$ & $0.02(0.02)$ & $0.06(0.03)$ & $0.02(0.02)$ \\
\hline & & \multicolumn{5}{|c|}{ Average size (Std. error) } \\
\hline 480 & 1 & $7.4(0.09)$ & $7.3(0.10)$ & $6.92(0.06)$ & $5.1(0.10)$ & $4.5(0.26)$ \\
\hline 1000 & 1 & $7.14(0.08)$ & $7.08(0.08)$ & $7(0.06)$ & $6.84(0.05)$ & $4.58(0.15)$ \\
\hline 2000 & 1 & $7.26(0.07)$ & $7.30(0.09)$ & $7(0.09)$ & $6.88(0.05)$ & $6.92(0.04)$ \\
\hline 3000 & 1 & $7.28(0.08)$ & $7.2(0.09)$ & $7.2(0.09)$ & $6.98(0.05)$ & $6.86(0.05)$ \\
\hline 480 & 2 & $6.84(0.08)$ & $7(0.05)$ & $6.88(0.07)$ & $5.16(0.14)$ & $4.7(0.13)$ \\
\hline 1000 & 2 & $6.96(0.06)$ & $6.98(0.05)$ & $6.94(0.07)$ & $6.9(0.04)$ & $4.58(0.15)$ \\
\hline 2000 & 2 & $6.82(0.05)$ & $6.86(0.05)$ & $6.90(0.05)$ & $6.82(0.08)$ & $6.86(0.05)$ \\
\hline 3000 & 2 & $6.82(0.05)$ & $6.82(0.05)$ & $6.86(0.06)$ & $6.82(0.05)$ & $6.9(0.04)$ \\
\hline 480 & 3 & $6.62(0.08)$ & $6.72(0.07)$ & $6.36(0.11)$ & $17.38(2.77)$ & $8.3(1.18)$ \\
\hline 1000 & 3 & $6.86(0.05)$ & $6.80(0.06)$ & $6.76(0.07)$ & $6.90(0.63)$ & $14.1(2.75)$ \\
\hline 2000 & 3 & $6.78(0.08)$ & $6.78(0.06)$ & $6.90(0.05)$ & $6.82(0.06)$ & $7.24(1.17)$ \\
\hline 3000 & 3 & $6.82(0.05)$ & $6.74(0.07)$ & $6.8(0.06)$ & $6.72(0.06)$ & $6.7(0.07)$ \\
\hline
\end{tabular}




\subsection{Source analysis on a real $M E G$ dataset}

We applied the proposed methodology to a human MEG dataset provided by Professor Richard Henson from the MRC Cognition and Brain Sciences Unit

540 Volunteer Panel (Henson et al., 2011). The study subject, a healthy young adult underwent the following face perception test which includes two different stimuli (faces and scrambled faces). A central fixation cross (presented for a random duration of 400 to $600 \mathrm{~ms}$ ) was followed by a face or scrambled face (presented for a random duration of 800 to $1000 \mathrm{~ms}$ ), followed by a central

${ }_{545}$ circles for $1700 \mathrm{~ms}$. As soon as he saw a face or a scrambled face, the subject used either their left or right index finger to report whether he thought it was symmetrical or asymmetrical vertically through its center. There were 96 trials labeled as Face and 50 labeled as Scrambled Face. The MEG data were collected with a VectorView system, containing a magnetometer and two orthogonal, planar gradiometers located at each of 102 positions within a hemispherical array situated in a light, magnetically shielded room. The sample rate was set at $1100 \mathrm{~Hz}$. Here, we investigated the dataset recorded by the 102 magnetometer. We want to identify voxels which showed power increases for the faces relative to the scrambled faces.

${ }_{555}$ We first created a grid system of $1 \mathrm{~cm}$ resolution with 1487 grid points, using the subject's anatomical magnetic resonance imaging (MRI) data. Then, we applied the neuroimaging software SPM8 to read and preprocess the recorded data, and to epoch and average the data over the trials for the face stimulus and the scrambled face stimulus respectively. This gives rise to 146 epochs of $700 \mathrm{~ms}$ (770 time instants) with $200 \mathrm{~ms}$ pre-stimulus and $500 \mathrm{~ms}$ post-stimulus. For each of the two stimulus, we calculated the sample covariance $\hat{C}$ from the post-stimulus data and the noise covariance $\hat{C}_{0}$ from pre-stimulus data. The signal-to-noise ratios for both datasets are close to 1 . We thresholded $\hat{C}$ by $c_{0} \hat{\sigma}_{0}^{2} \sqrt{\log (n) / J}$, where $n=102, J=551$, and $\hat{\sigma}_{0}^{2}$ is the minimum diagonal element in $\hat{C}_{0}$. We only reported the results when setting $c_{0}=0.5$, since the results are similar for other values of $c_{0}$.

After the pre-processing above, we performed the proposed SAM procedure 
on the face dataset and the scrambled face dataset respectively and calculated the logarithm of the SAM index ratio (i.e., the log-contrast) for each of 1487 grid the above observed log-ratios, as we note that the baseline distribution at each grid point can spatially vary. To adjust for such a spatial heterogeneity, we first generated an empirical baseline distribution by randomly partitioning the 196 trials into two groups sized 96 and 50 respectively. We labeled the two groups repeated 80 times, producing 80 paired datasets. We then calculated the SAM log-ratios for these paired datasets respectively, which generated an empirical baseline distribution for each grid. In Figure 1, we plotted the observed logcontrasts against the baselines, suggesting a striking difference between them.

Finally, we adjusted the observed log-ratio for each grid by subtracting the corresponding empirical baseline mean. In Figure 1, we also plotted the adjusted log-ratios on 20 traverse slices and on the three orthogonal slices through the maximum location estimate. The plots suggest two regions of the SAM-index increasing, which are around the grid points $(0,6,6)$ and $(-2,6,5)$ respectively.

In Figure 1, the scree plot in the top-left clearly suggests that a change-point might have occurred at the ordered slice 5. Therefore, we used the peak 0.0698 on this slice to estimate the threshold for screening. The screening provides a source set containing 22 grid points, where the SAM index shows increases for the faces relative to the scrambled faces. The estimated time-courses at $(0,6,6)$ and $(-2,6,5)$ plotted in Figure 1 show large differential responses of the brain to the faces and the scrambled faces at these two positions. These results have not been found by Henson et al.(2011) via a parametric Bayesian approach.

\section{Discussion and conclusion}

The beamformer-based screening has been widely used by neuron-scientists 595 In this paper, we have reformulated the problem as finding sparse coefficients 
in a time-varying coefficient model. Therefore, the proposed procedure is immediately applicable to estimating sparse time-varying coefficient models. We have improved the commonly used screening procedure by adding a data-driven choice of threshold. We have proved the sure screening property for the proposed procedure under some regularity conditions. We have also provided asymptotic bounds for screening errors in the two-source setting when both the number of sensors and the sampling rate are tending to infinity. To assess the finite sample performance of the proposed screening procedure, we have conducted simula${ }_{605}$ tion studies. The simulation results have suggested that the proposed screening procedure is promising in detecting sources of moderate signal-to-noise ratios.

The aim of beamforming is to allow the neuronal signal of interest to pass through in certain source location and orientation, while suppressing noise or unwanted signal in other source location or orientation. In practice, the unwanted signals from other locations cannot be fully blocked. Therefore, the source interference may prevent one from localizing the underlying sources. The existing theoretical analysis on the above issue was based on the unrealistic assumption that the sensor population covariance matrix is known (e.g., Sekihara and Nagarajan, 2010). In general, there is lack of a statistically sounding theory which allows one to address: (1) how to conduct a sure screening on a beamformer map, (2) how the spatial and temporal dimensions determine the accuracy of the estimated sources derived from the screening. Here, we have offered a theoretical analysis based on the estimated sensor covariance matrix in which we have allowed both the number of sensors and the sampling rate to be varying. The new analysis has drawn a clear picture on how the spatial and temporal dimensions are related to the accuracy of the beamformer-based screening. We have illustrated the proposed procedure by using a real MEG neuroimage analysis. Two interesting theoretical topics remain untouched: one is on the asymptotic behavior of the proposed data-driven procedure for choosing the threshold and the other is on determining the number of sources in a neuroimage. However, extending the theory to cover these two topics is beyond the scope of the paper. 


\section{Appendix}

In the Appendix, suppressing $c_{0}$ in $\operatorname{SAM}_{c_{0}}(r)$, we let $\operatorname{SAm}(r)$ denote $\operatorname{SAm}(r)$. ${ }_{630}$ Note that $c_{0}$ will not affect the convergence rate of the index.

Lemma 6.1. For any lead field vectors $\mathbf{x}, \mathbf{x}_{1}$, and $\mathbf{x}_{2}$, we have

$$
\left(\rho\left(\mathbf{x}, \mathbf{x}_{2}\right)-\rho\left(\mathbf{x}, \mathbf{x}_{1}\right) \rho\left(\mathbf{x}_{1}, \mathbf{x}_{2}\right)\right)^{2} \leq\left(1-\rho\left(\mathbf{x}, \mathbf{x}_{1}\right)^{2}\right)\left(1-\rho\left(\mathbf{x}_{1}, \mathbf{x}_{2}\right)^{2}\right) .
$$

The two sides are equal if and only if $\mathbf{x}$ is a linear combination of $\mathbf{x}_{1}$ and $\mathbf{x}_{2}$.

In the following lemmas, for simplicity, let $\hat{C}_{2}$ denote $\hat{C}\left(\tau_{n J}\right)$, where $\tau_{n J}=$ $O(n \sqrt{\log (n) / J})$.

${ }_{635}$ Lemma 6.2. Under conditions $(A 1) \sim(A 5)$, if $(\log (n))^{\kappa_{3}} / J=o(1)$ and $n \sqrt{\log (n) / J}=$ $o(1)$, then we have

$$
\left|\frac{\mathbf{x}^{T} \hat{C}_{2}^{-1} \mathbf{x}}{\mathbf{x}^{T} \hat{C}_{2}^{-2} \mathbf{x}}-\frac{\mathbf{x}^{T} C_{2}^{-1} \mathbf{x}}{\mathbf{x}^{T} C_{2}^{-2} \mathbf{x}}\right|=O_{p}(n \sqrt{\log (n) / J}),
$$

$n$ and $J$ tend to infinity.

In the following lemma, for $k=1,2$, we investigate the behavior of the SAM index in an $O\left(n^{-1}\right)$-neighborhood of $r_{k}$ but not in an $O\left(1 /\left(n \psi_{n}\right)\right)$-lead field ${ }_{640}$ neighborhood of $r_{k}$. For this purpose, for any positive constants $a_{n} \rightarrow \infty$ and $b_{n}=O(1)$, define

$$
\Phi_{11 \mid k}=\left\{\mathbf{x} \in \Phi: \psi_{n} \zeta_{n}(\mathbf{x} \mid k) \geq a_{n}, \zeta_{n}(\mathbf{x} \mid k) \leq b_{n}\right\}, k=1,2
$$

Lemma 6.3. Under conditions (A1) (A6), if $\psi_{n} \rightarrow \infty$, then

$$
\sup _{\mathbf{x} \in \Phi_{1 \mid k}} \frac{\mathbf{x}^{T} C_{2}^{-1} \mathbf{x}}{\mathbf{x}^{T} C_{2}^{-2} \mathbf{x}} \leq \sigma_{0}^{2}\left(1+\frac{\sigma_{0}^{2}}{\gamma_{k} \zeta_{n}(\mathbf{x} \mid k)}\right)|O(1)|
$$

as $n$ and $J$ tend to infinity. 
For any constant $a_{n} \rightarrow \infty$, we consider a set outside neighborhoods of $r_{1}$ 645 and $r_{2}$, defined by

$$
\Phi_{2}=\left\{\mathbf{x} \in \Phi: \zeta_{n}(\mathbf{x} \mid 1) \geq a_{n}, \zeta_{n}(r \mid 2) \geq a_{n}\right\}
$$

We have the following lemma.

Lemma 6.4. Under conditions (A1) (A6), if $\psi_{n} \rightarrow \infty$, then uniformly for $\mathbf{x} \in \Phi_{2}$

$$
\frac{\mathbf{x}^{T} C_{2}^{-1} \mathbf{x}}{\mathbf{x}^{T} C_{2}^{-2} \mathbf{x}}=\sigma_{0}^{2}\left(1+O\left(1+O\left(\frac{1}{n}+\frac{1}{\psi_{n}^{2}}\right)\right)\right)
$$

as $n$ and $J$ tend to infinity.

To examine the behavior of the SAM index in the $O\left(1 / n^{2}\right)$ neighborhood of $r_{k}$, for any positive constant $b_{n}=O(1)$, we consider the neighborhood of $r_{k}$ defined by

$$
\Phi_{3 \mid k}=\left\{\mathbf{x} \in \Phi: \psi_{n} \zeta_{n}(\mathbf{x} \mid k) \leq b_{n}\right\}, k=1,2 .
$$

Lemma 6.5. Under conditions $(A 1) \sim(A 6)$, if $\psi_{n} \rightarrow \infty$, then uniformly for $\mathrm{x} \in \Phi_{3 \mid 1}$

$$
\frac{\mathbf{x}^{T} C_{2}^{-1} \mathbf{x}}{\mathbf{x}^{T} C_{2}^{-2} \mathbf{x}}=\frac{\gamma_{1} \psi_{n}\left(1+O\left(1 / \psi_{n}\right)\right)}{1+\gamma_{1}^{2}\left(\psi_{n} \zeta_{n}(\mathbf{x} \mid 1)-\delta_{n}(\mathbf{x} \mid 12)^{2}\right) / \sigma_{0}^{4}}
$$

as $n$ tends to infinity. The similar result holds uniformly for $\mathbf{x} \in \Phi_{3 \mid 2}$.

\section{Proof of Theorem 3.1: Let}

$$
\begin{aligned}
S_{11 \mid 1}^{c} & =\left\{r \in \Omega: \psi_{n} \zeta_{n}(r \mid 1)>a_{n}, \zeta_{n}(r \mid 1)=O(1)\right\}, \\
S_{12 \mid 1}^{c} & =\left\{r \in \Omega: \psi_{n} \zeta_{n}(r \mid 1)>a_{n}, \zeta_{n}(r \mid 1) \rightarrow \infty\right\}, \\
S_{11 \mid 2}^{c} & =\left\{r \in \Omega: \psi_{n} \zeta_{n}(r \mid 2)>a_{n}, \zeta_{n}(r \mid 2)=O(1)\right\}, \\
S_{12 \mid 2}^{c} & =\left\{r \in \Omega: \psi_{n} \zeta_{n}(r \mid 1)>a_{n}, \zeta_{n}(r \mid 2) \rightarrow \infty\right\} .
\end{aligned}
$$

Then

$$
\left(S_{1 \mid 1} \cup S_{1 \mid 2}\right)^{c}=S_{1 \mid 1}^{c} \cap S_{1 \mid 2}^{c} \subseteq S_{11 \mid 1}^{c} \cup S_{11 \mid 2}^{c} \cup\left(S_{12 \mid 1}^{c} \cap S_{12 \mid 2}^{c}\right) .
$$


In the following, we show that

$$
\begin{aligned}
& P\left(D_{n} \cap S_{11 \mid 1}^{c}=\emptyset\right) \rightarrow 1, \quad P\left(D_{n} \cap S_{11 \mid 2}^{c}=\emptyset\right) \rightarrow 1, \\
& P\left(D_{n} \cap S_{12 \mid 1}^{c} \cap S_{12 \mid 2}^{c}=\emptyset\right) \rightarrow 1,
\end{aligned}
$$

respectively.

By Lemmas 6.2 and 6.3, we have for any constant $c$, with a probability tending to one, uniformly for $r \in S_{11 \mid 1}^{c}$,

$$
\begin{aligned}
\operatorname{sAM}(r) & \leq \sigma_{0}^{2}\left(1+\frac{\sigma_{0}^{2}}{\gamma_{1} \zeta_{n}(r)}\right)|O(1)|+c(n \sqrt{\log (n) / J}) \\
& \leq \sigma_{0}^{2}\left(1+\frac{\sigma_{0}^{2} n}{\gamma_{1} a_{n}}\right)|O(1)|+c(n \sqrt{\log (n) / J}),
\end{aligned}
$$

which is smaller than $\sigma_{0}^{2}\left(1+h_{n}\right)$ by the definition. Therefore, $r \notin D_{n}$ when $n$ and $J$ are large enough. This implies $P\left(D_{n} \cap S_{11 \mid 1}^{c}=\emptyset\right) \rightarrow 1$. Similarly, we can show that $P\left(D_{n} \cap S_{11 \mid 2}^{c}=\emptyset\right) \rightarrow 1$.

By Lemmas 6.2 and 6.4, we have for any constant $c$, with a probability tending to one, uniformly for $r \in S_{12 \mid 1}^{c} \cap S_{12 \mid 2}^{c}$,

$$
\begin{aligned}
\operatorname{sAM}(r) & \leq \sigma_{0}^{2}(1+o(1))+c(n \sqrt{\log (n) / J}) \\
& <\sigma_{0}^{2}\left(1+h_{n}\right),
\end{aligned}
$$

as $n$ and $J$ tend to infinity. This yields

$$
P\left(D_{n} \cap S_{12 \mid 1}^{c} \cap S_{12 \mid 2}^{c}=\emptyset\right) \rightarrow 1 .
$$

For $r \in S_{2 \mid 1}$, without loss of generality, we assume that $\psi_{n} \zeta_{n}(r)=\zeta+o(1)$ and $n \delta_{n}(r)=\delta+o(1)$. We have

$$
\left|\rho\left(\hat{\mathbf{x}}, \mathbf{x}_{2}\right)-\rho_{12}\right| \leq \sqrt{2\left(1-\rho\left(\hat{\mathbf{x}}(r), \mathbf{x}_{1}\right)\right)}=O(1 / n) .
$$

By Lemmas 6.2 and 6.5, for any constant $c>0$, with a probability tending to one, uniformly for $r \in S_{2 \mid 1}$, we have

$$
\operatorname{SAM}(r) \geq \frac{\gamma_{1} \psi_{n}}{1+\zeta \gamma_{1}^{2} / \sigma_{0}^{4}+o(1)}+c(n \sqrt{\log (n) / J})
$$

which is larger than $\sigma_{0}^{2}\left(1+h_{n}\right)$ when $h_{n} / \psi_{n}=o_{p}(1)$. Therefore, $r \in D_{n}$. This yields $P\left(S_{2 \mid 1} \subseteq D_{n}\right) \rightarrow 1$. Similarly, we can show that $P\left(S_{2 \mid 2} \subseteq D_{n}\right) \rightarrow 1$.

The proof is completed. 


\section{Acknowledgements}

We thank Professor Richard Henson for sharing with us his MEG neuroimaging data.

[1] Bickel, P. and Levina, E., 2008. Covariance regularization by thresholding. Ann. Stat., 36, 2577-2604.

[2] Brookes, M.J., Vrba,J., Robinson,S.E., Stevenson, C.M., Peters, A.M., Barnes, G.R., Hillebrand, A. and Morris,P.G.,2008. Optimizing experimental design for MEG beamformer imaging. NeuroImage, 39, 1788-1802.

[3] Fan, J. and Li, R., 2001. Variable selection via nonconcave penalised likelihood and its oracle properties. Jour Ameri Statist Assoc, 96, 1348-1360.

[4] Fan, J. and Lv, J., 2008. Sure independence screening for ultra-high dimensional feature space (with discussions). J our Roy Statist Soc B , 70, 849-911.

[5] Hamalainen, M., Hari, R., Ilmoniemi,R.J., Knuutila, J. and Lounasmaa, O.V., 2010. Magnetoencephalography theory, instrumentation, and applications to noninvasive studies of the working human brain. Rev. Modern Phys., 21, 413-460.

[6] Henson, R.N., Wakeman,D.G., Litvak,V. and Friston,K,J., 2011. A parametric em- pirical Bayesian framework for the EEG/MEG inverse problem: generative models for multi-subject and multi-modal integration. Front. Hum. Neuroscience, $5,1-16$.

[7] Meinshausen, N. and Bühlmann, P., 2006. High dimensional graphs and variable selection with the Lasso. Ann. Statist., 34, 1436-1462.

[8] Quraan, M.A., Moses,S.N., Hung,Y., Mills, T. and Taylor, M.J., 2011. Detection and localization of hippocampal activity using beamformers with MEG: 
A detailed investigation using simulations and empirical data. Human Brain Mapping, 32, 812- 827.

[9] Robinson, S. and Vrba, J., 1998. Functional neuroimaging by synthetic aperture magnetometry. In: Recent Advances in Biomagnetism. Yoshimoto, T., Kotani, M., Kuriki, S. Karibe, H., Nakasato, N. (Eds.), Tohoku University Press, Sendai, Japan, pp. 302-305.

[10] Sarvas, J., 1987. Basic mathematical and electromagnetic concepts of the biomagnetic inverse problem. Phys. Med. Biol., 32, 11-22.

[11] Sekihara, K. and Nagarajan, S.S., 2010. Adaptive Spatial Filters for Electromagnetic Brain Imaging. Springer-Verlag, Berlin.

[12] Tibshirani, R., 1996. Regression shrinkage and selection via the Lasso. Jour Roy Statist Soc B, 58, 267-288.

[13] van Veen, B.D., van Drongelen, W., Yuchtman, M. and Suzuki, A., 1997. Localization of brain electrical activity via linearly constrained minimum variance spatial filtering. IEEE Trans. Biomed. Eng., 44, 867-880.

[14] Tibshinari,R., Walther, J. and Hastie, T., 2001. Estimating the number of clusters in a data set via the gap statistic. Jour. Roy. Stat. Soc. B, 63, $411-423$.

[15] Zhang, C.H., 2010. Nearly unbiased variable selection under minimax concave penalty. Ann Statist., 38, 894-942.

[16] Zhang,J., Liu, C. and Green, G., 2014. Source localization with MEG data: A beamforming approach based on covariance thresholding. Biometrics , 70, $121-131$.

[17] Zhao, P. and Yu, B., 2006. On model selection consistency of LASSO. Jour Machine Learning Research, 7, 2541-2567. 

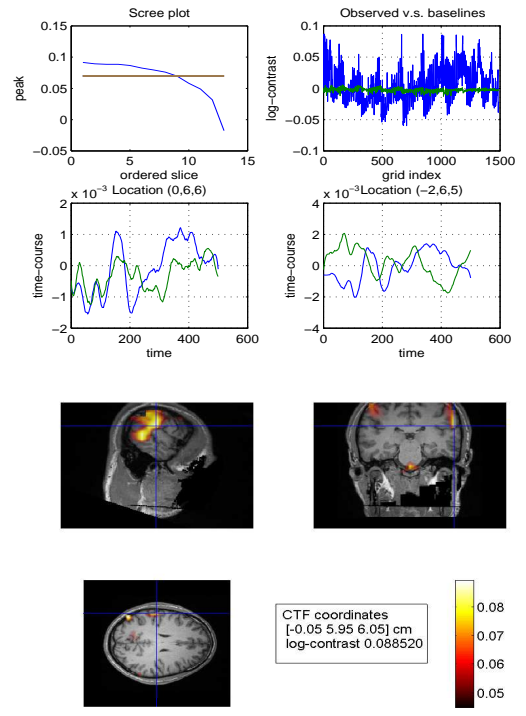
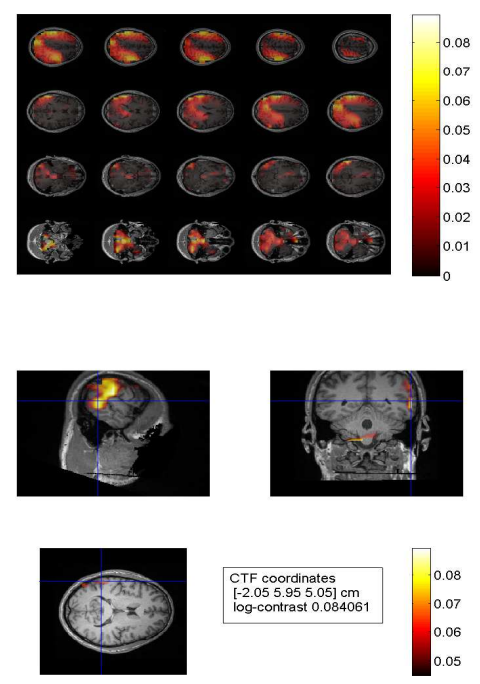

Figure 1: The top row in the left two columns: The plot in the left is a scree plot for the peaks of the adjusted SAM log-contrasts against the ordered slices, where the yellow-colored line indicates the estimated threshold level. The plot in the right provides a comparison between the observed log-contrasts and the baselines. The second row in the left two columns: The plots from left to right show the estimated time-courses at the two estimated source locations, where the face and the scrambled time-courses are colored by blue and green respectively. The remaining plots are derived from the adjusted SAM log-ratio-based mapping for the faces relative to the scrambled faces. The upright plots show the adjusted SAM log-ratios on 20 transverse slices evenly distributed from the top to bottom along the z-axis, where the upper, middle and bottom panels show the map on the slices along the top-to-bottom direction of the z-axis respectively. The $(i, j, k)$ stands for the coordinates of grid point $\left(r_{x}, r_{y}, r_{z}\right)$ in the CTF system of the brain. See the software FieldTrip for the definition. The bottom two rows: The adjusted SAM log-ratio-based maximum-mapping for scrambled faces relative to faces. The plots show the adjusted SAM log-ratios the three orthogonal slices through the locations $(0,6,6) \mathrm{cm}$ and $(-2,6,5) \mathrm{cm}$ respectively. In the plots, the adjusted log-contrasts are increasing in the color order from blue to light blue to green to light yellow to dark red. 